\title{
MÉTODO PARA CONTORNAR O PROBLEMA DE ESTIMA- TIVAS NEGATIVAS EM COMPONENTES DE VARIÂNCIA
}

\author{
CESAR PEREIRA
}

Orientador: Prof. Dr. IZAIAS RANGEL NOGUEIRA

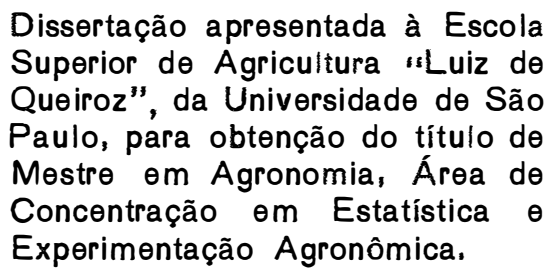

$P|R A C| C A B A$

Estado de São Paulo - Brasil

Maio, 1983 
ii.

Aos meus pais,

Cecilia e oscar (in memorian)

A minha filha,

Marice,

Aos meus irmãos,

Ana Maria, Oscar e Silvana,

A minha companheira,

Zeza,

DEDICO. 
iii.

\section{AGRADECIMENTOS}

Ao Prof. Dr. Izaias Rangel Nogueira, pela eficiente orientação.

Ao Prof. Dr. Décio Barbin, pelas sugestões, colabora ção e revisão do texto.

Ao Prof. Dr. Humberto de Campos, Chefe do Departamen to de Matemática e Estatística e Coordenador do Curso de Pós-Gradua ção em Estatística e Experimentação Agronômica da ESALQ/USP.

Aos Professores e Funcionários do Departamento de Ma temātica e Estatística da ESALQ, pela dedicação e colaboração.

Aos colegas do Curso de Estatística e Experimentação Agronômica, pela amizade e colaboração prestada.

$\bar{A}$ Fundação Universidade Estadual de Maringá, pela oportunidade.

A CAPES, através do PICD, pela bolsa de estudo concedida.

A todos que, de uma forma ou de outra, colaboraram pa ra a execução deste trabalho. 


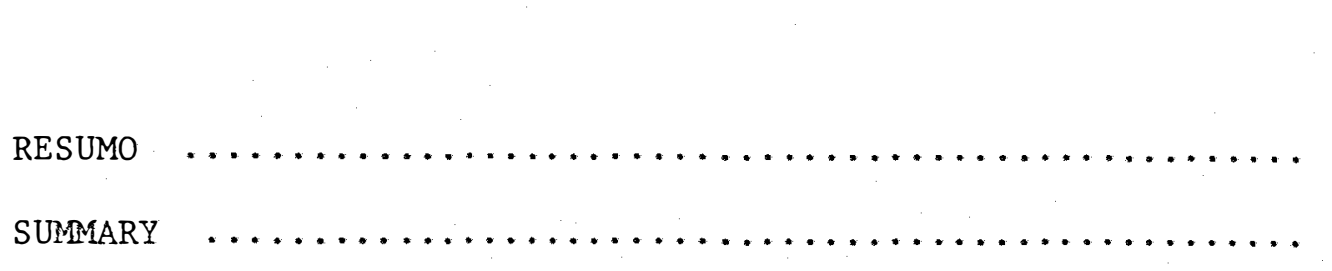

Pāg.

vi

viii

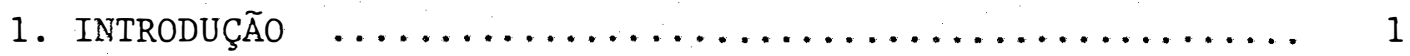

2. REVISÃo DE Literatura $\ldots \ldots \ldots \ldots \ldots \ldots \ldots \ldots \ldots \ldots \ldots \ldots \ldots \ldots \ldots \ldots \ldots \ldots$

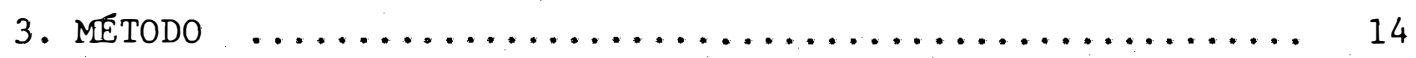

3.1 - Modelo Matemático $\ldots \ldots \ldots \ldots \ldots \ldots \ldots \ldots \ldots \ldots \ldots \ldots \ldots$

3.1 .1 - Modelo aleatório (Tipo II) ........... 17

3.1.1.1 - Somas de quadrados ......... 19

3.1.1.2 - Determinação das esperanças matemā

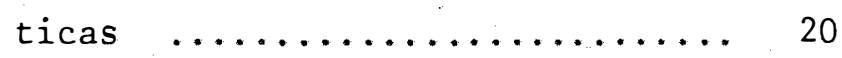

3.1.1.3 - Esperança matemática do quadrado mē dio dos fatores $\ldots \ldots \ldots \ldots \ldots 27$

3.1.1.4 - Quadro de análise de variância . 30

3.1 .1 .5 - Estimativas de $\rho, \hat{\sigma}_{\mathrm{a}}$ e $\sigma^{2} \ldots \ldots \ldots 31$

3.1 .1 .6 - Valores de a, b e c ........ 33

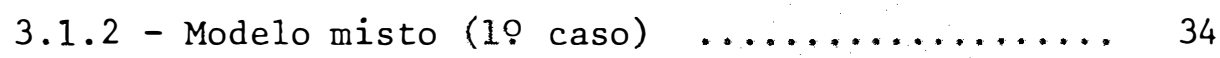

3.1 .2 .1 - Somas de quadrados ........ 35

3.1.2.2 - Determinação das esperanças matemá ticas $\ldots \ldots \ldots \ldots \ldots \ldots \ldots \ldots . \ldots \ldots$

3.1.2.3 - Esperança matemática do quadrado mé dio dos fatores $\ldots \ldots \ldots \ldots . \ldots 40$

3.1.2.4 - Quadro de anảlise de variância ... 42

3.1 .2 .5 - Estimativas de $\rho$ e $\sigma^{2} \ldots \ldots \ldots \ldots 43$ 
Pàg.

3.1 .3 - Modelo misto $(2 \circ$ caso $) \quad \ldots \ldots \ldots \ldots \ldots \ldots 44$ 3.1 .3 .1 - Somas de quadrados $\ldots \ldots \ldots \ldots 46$

3.1 .4 - Modelo fixo (tipo I) ............. 46

3.1 .4 .1 - Somas de quadrados .......... 48

4. EXEMPLO NUMERICO ........................ 49

5. RESULTADOS OBtidDOS .......................... 54

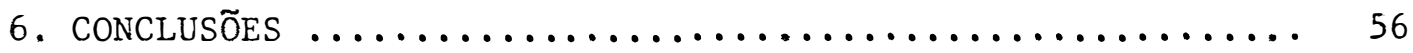

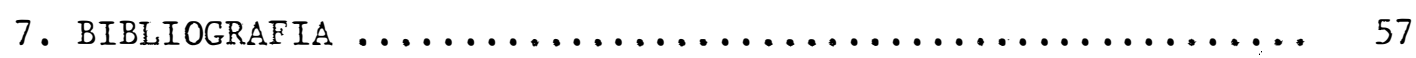


METODO PARA CONTORNAR O PROBLEMA DE ESTIMATIVAS NEGATIVAS EM COMPONENTES DE VARIÃNCIA

Autor: Cesar Pereira

Orientador: Prof. Dr. Izaias Rangel Nogueira

Numa análise de variância, a presença de correlação negativa entre os elementos de uma mesma parcela (correlação intra-classe) pode ocasionar estimativas negativas de componentes de variância no modelo de classificação hierārquica.

0 modelo matemático considerado foi:

$$
y_{i j k}=\mu+a_{i}+b_{i j}+e_{i j k} .
$$

Ao fazermos uma análise de variância no modelo de classificação hierárquica, considerando este modelo aleatōrio, misto (dois casos) ou fixo, se determinarmos uma estimativa de compo nente de variância negativo e pelo teste $F$ bilateral a hipōtese de nulidade for rejeitada ao nỉvel $\alpha$ de significância, eliminamos o pa 
vii.

râmetro correspondente no modelo matemätico.

$$
\begin{gathered}
\text { Então o modelo } y_{i j k}=\mu+a_{i}+b_{i j}+e_{i j k} \text { passa para } \\
y_{i j k}=\mu+a_{i}+e_{i j k}
\end{gathered}
$$

e reestruturamos a anālise de variância, supondo

$$
E\left[e_{i j k} \cdot e_{i} j^{\prime} k^{\prime}\right]=\left\{\begin{array}{l}
\sigma^{2}, \text { para } i=i^{\prime}, j=j^{\prime} \text { e } k=k^{\prime} \\
\rho \sigma^{2}, \text { para } i=i^{\prime}, j=j^{\prime} \text { e } k \neq k^{\prime} \\
0, \text { para os demais casos }
\end{array}\right.
$$

compomos um novo quadro de análise de variância a partir das somas de quadrados e respectivas esperanças matemáticas, através do método dos momentos.

A partir do novo quadro de análise de variância esti mamos os novos componentes de variância e a correlação intra-classe. 
viii.

METHOD TO SOLVE THE PROBLEM OF NEGATIVE ESTIMATIVES

IN COMPONENTS OF VARIANCE

Author: Cesar Pereira

Adviser: Prof. Dr. Izaias Rangel Nogueira

$S U M M A R Y$

The main purpose of the present work is to determine the new estimatives of the components of variance when its negative estimatives occur in the standard or hierarchical classification considering the following pattern: random, mixed or fixed, and the estimatives occur in the standard or hierarchical classification.

This mathematical pattern was considered:

$$
y_{i j k}=\mu+a_{i}+b_{i j}+e_{i j k} .
$$

When we make an analysis of variance in the hierarchical classification considering the pattern shown above, if we determine a estimative of component of negative variance and by the bilateral $F$ test, the null hypothesis is rejected at the $\alpha$ zeyel of meaning we eliminated the corresponding parameter in the mathematical model. 


$$
\begin{gathered}
\text { So the nodel } y_{i j k}=\mu+a_{i}+b_{i j}+e_{i j k} \text { becomes } \\
y_{i j k}=\mu+a_{i .}+e_{i j k},
\end{gathered}
$$

and we re-structure the analysis of variance, supposing:

$$
\mathbb{E}\left[e_{i j k} \cdot e_{i} j^{\prime} k^{\prime}\right]=\left\{\begin{array}{cc}
\sigma^{2}, \text { to } i=i^{\prime}, j=j^{\prime} \text { and } k=k^{\prime} \\
\rho \sigma^{2}, \text { to } i=i^{\prime}, j=j^{\prime} \text { and } k \neq k^{\prime} \\
0, \text { other cases }
\end{array}\right.
$$

we compose a new board of analysis of variance from the addition of same numbers and respective mathematical expectations across method of moments.

From the new board of the analysis of variance we es timate the new components of variance and the into-class correlation. 
1. INTRODUÇAO

Devido ao inconveniente do fato de estimarmos como negativo certos componentes de variância, o problema de estimativas negativas tem merecido por parte dos estatisticos um tratamento especial. Talvez não seja exagero afirmarmos que este fato tenha sido o responsāvel pelas recentes contribuições na área de componen tes de variância.

Uma abordagem para o problema de estimativas negativas, e mais fundamentada, é sugerir métodos alternativos de estimação que não produzem estimativas negativas para os componentes de variância.

Na tentativa de contornar esse problema, talvez como primeiro trabalho, surgiu o de HERBACH (1959), que usa estimadores de máxima verossimilhança, como vantagem o fato de serem não negati vos, e como desvantagem o fato de serem truncados e viciados.

THOMPSON (1962) na tentativa de resolver o problema de estimativas negativas desenvolveu um algoritmo, por ele denomina 
do de "pool-the-minimum-violator". Esse algoritmo resulta da maximização da função de verossimilhança dos quadrados médios, sujeita a um conjunto de restrições, que recebe a denominação de princípio de máxima verossimilhança restritivo. Este procedimento provoca um truncamento, e o estimador resultante è viciado. Através de HILL (1965) e TIAO \& TAN (1965) surgiram os primeiros trabalhos nesta ārea, tratando de inferência bayesiana sobre componentes de variância, num modelo aleatório simples. A grande vantagem prática do uso dos métodos bayesianos, é que as estimativas dos componentes de variância são sempre não negativas.

Numa anālise de variância, a presença de coirrelação negativa entre os elementos de uma mesma parcela (correlação intraclasse) pode ocasionar estimativas negativas de componentes de variância.

SEARLE (1971) enumera vārios procedimentos alternati vos, quando encontramos uma estimativa negativa de um componente de variância, os quais faremos as seguintes considerações:

I) Aceitamos a estimativa negativa, admitindo que o verdadeiro valor do componente de variância é zero. Isto pode pare cer lógico, mas como parte da estimação foi truncada, isto perturba as propriedades dos estimadores.

II) Sendo negativa uma estimativa de componente de variância, devemos eliminar o parâmetro correspondente no modelo, e reestruturamos a anālise de variância obtendo uma ponderação dos 
quadrados médios restantes, e, com isso, obtemos novas estimativas dos componentes de variância restantes.

Um problema que tem ocorrido ao aceitar a estimativa negativa (consideração I) é que, muitas vezes, valores negativos muito distantes de zero são tomados como tal. E aconselhável testar a hipótese nula utilizando o teste $F$ bilateral.

O objeto deste trabalho, é obtermos as novas estimativas dos componentes de variância através do método dos momentos, em classificação hierärquica, quando determinamos uma estimativa ne gativa do componente de variância nos modelos: aleatōrio, misto e fixo, e determinar a expressão da estimativa da correlação negativa entre as variāveis. 


\section{REVISAOO DE LITERATURA}

KING e HENDERSON (1954) apresentam em detalhes um mé todo para estimação dos componentes de variância de dados em classi ficação hierärquica com números desiguais dentro de sub-classes, ob tendo coeficientes dos componentes de variância.

SNEDECOR (1956), exemplificando um caso de classificaçäo hierärquica com os dados distribuídos em classes de: machos, fêmeas dentro de machos, e filhos dentro de uma mesma fêmea, obteve um quadrado médio para machos menor que o quadrado médio para fêmeas. Diz que isto não pode acontecer na populáção mesmo quando o componente para machos for igual a zero e sugere métodos alternativos de amostragem que devem ser. feitas para diminuir o verdadeiro quadrado médio para machos, ou aumentada a correspondente para fêmeas, ou ambas as coisas, e conclui ainda que está claro que não há 
evidência conträria ao fato de se admitir o. componente de variância para macho como igual a zero.

SNEDECOR (1956) apresenta um método aproximado para determinar os coeficientes dos componentes de variância para dados dispostos numa classificação hierärquica com sub-classes de tamanhos diferentes, desde que essas desigualdades não sejam muito gran des.

$$
\begin{aligned}
& \text { Esses coeficientes são assim determinados: } \\
& \qquad k_{1}=\frac{1}{N_{d}-1}\left(N-\frac{j n_{j}^{2}}{N}\right) ;
\end{aligned}
$$

onde:

$$
\begin{aligned}
k_{1} & =\text { nümero aproximado de descendentes por fêmea; } \\
N & =\text { nümero total de descendentes; } \\
N_{d} & =\text { nümero de fêmeas; } \\
n_{j} & =\text { nümero de descendentes de cada fêmea; } \\
k_{2} & =\frac{1}{N_{s}-1}\left(N-\frac{i}{N}\right)
\end{aligned}
$$

onde: $k_{2}=$ nümero a proximado de descendente por macho;

$$
\begin{aligned}
\mathrm{N} & =\text { nümero total de descendentes; } \\
\mathrm{N}_{\mathrm{s}} & =\text { nümero de machos; } \\
\mathrm{n}_{\mathrm{i}} & =\text { nümero de descendentes de cada macho. }
\end{aligned}
$$


Afirma que essa utilização permite a aplicação de um teste $\mathrm{F}$ exato, baseado nos valores aproximados dos coeficientes dos componentes.

COCHRAN e COX (1957) admitem a possibilidade do fato de que observações entre diferentes sub-unidades de uma mesma unida de experimental podem estar correlacionadas. Citam que em experimen tos de campo esta correlação è um reflexo do fato de que porções de terra vizinhas tendam a ser semelhantes quanto à fertilidade e outras propriedades agronômicas. Mostram que a variância aplicável às sub-unidades é $\sigma^{2}(1-\rho)$, e às unidades è $\sigma^{2}[1+(\beta-1) \rho]$, sendo: $\sigma^{2}=$ = variância entre sub-unidades de uma mesma unidade; $\rho$ = coeficien te de correlação e $\beta=$ número de sub-unidades dentro de cada unidade.

FALCONER (1960) salienta que, se houver números desi guais de filhos por fêmea ou de fêmeas por machos, pode-se utilizar como coeficiente de $\sigma_{\mathrm{D}}^{2}$ o número médio de descendente por fêmea e co mo coeficiente $\sigma_{\mathrm{S}}^{2}$ o nümero médio de fêmeas por macho. Isso traria pouco erro, desde que a desigualdade dos números não fosse muito grande.

STEEL e TORRIE (1960) citam a existência de uma correlação intra-classe que é estimada por:

$$
r_{I}=\frac{Q M(\text { Entre Classes) }-Q M \text { (Dentro de Classes) }}{Q M(\text { Entre Classes) }+(n-1) Q M \text { (Dentro de Classes) }} \text {, }
$$

onde $\mathrm{n}=$ nümero de observações por classe. 
0 mesmo resultado seria obtido da equação:

$$
r=\frac{\hat{\sigma}_{r}^{2}}{\hat{\sigma}_{r}^{2}+\hat{\sigma}^{2}}
$$

onde $\hat{\sigma}^{2}$ é a estimativa do componente de variância dentro de classes e $\hat{\sigma}_{r}^{2}$ é a estimativa do componente de variância entre classes. Afirmam que isto é vālido mesmo quando o número de observações varia de uma classe para outra. Indicam que um teste de significância para a correlação intra-classe é o teste F. 0 teste deve ser feito usando as variâncias entre e dentro de classes no numerador e denominador, respectivamente. Aconselham, então, fazer um teste de significância antes de calcular a correlação. Indicam, ainda, que quando o quadrado médio entre classes é menor que o quadrado médio dentro de classes, deve-se tomar a estimativa $\sigma_{r}^{2}$ como zero, isto $\bar{e}, \quad \hat{\sigma}_{r}^{2}=0$. Mostram, que $r_{I}$ pode variar de

$$
+1 \text { até }-\frac{1}{n-1} \text {, }
$$

que $\overline{\mathrm{e}}$ o caso quando QM Entre Classes $=0$.

KALIL (1963) estudou herdabilidade do período de ser viço de vacas Gir e Guzerá pelo método de correlação entre meio-irmãos paternos, com as causas de variação: Raças, Touros dentro de Raças, Meios-irmãs paternas, com respectivamente 1, 6 e 141 graus de liberdade.

Encontrou uma estimativa de variância para Touros $\hat{\sigma}_{\mathrm{T}}^{2}=-0,13$ 
Concluiu, como uma variância negativa è impossível por definição, a interpretação mais plausível dessa estimativa é atribuir o sinal negativo a erro de amostragem e considerā-la como sendo uma estimativa de variância nula.

PANSE e SUKHATME (1963) citam exemplos para ilustrar a utilidade da correlação intra-classe. Afirmam que um valor positi vo da correlação intra-classe significa que a variação dentro do gru po $\vec{e}$ menor que a entre os grupos, enquanto que um valor negativo do coeficiente significa que as médias dos grupos tendem a ser semelhantes, apesar da variação dentro dos grupos. Um valor próximo de zero mostraria que as variações dentro dos grupos e entre eles são da mesma ordem, isto é, que a variação real entre grupos não é maior nem menor que dentro dos grupos.

PIMENTEL GOMES et alii (1964), trabalhando com amostragem de cana-de-açūcar para determinações tecnológicas, utilizaram, para cada variedade ensaiada, 2 blocos, 15 touceiras dentro de cada bloco e 4 canas dentro de cada touceira.

Consideraram inicialmente o modelo matemático;

$$
y_{i j k}=m+b_{i}+t_{i j}+e_{i j k} \text {, }
$$

onde,

$$
\begin{aligned}
\mathrm{m} & =\text { mëdia da variedade; } \\
\mathrm{b}_{i} & =\text { efeito do bloco (fixo) } \underline{i}(i=1,2,) ; \\
t_{i j} & =\text { efeito (aleatório) da touceira } \underline{j} \text { no bloco } \underline{i}(j=1,2, \ldots, 15) ; \\
e_{i j k} & =\text { efeito (aleatório) da cana } \underline{k} \text { na touceira } \underline{j} \text { do bloco } \underline{i}(k= \\
& =1,2,3,4) .
\end{aligned}
$$


A anālise da variância apresentou o esquema:

\begin{tabular}{l|c|c|c}
\hline Causas de Variação & G.L. & Q.M. & E(Q.M.) \\
\hline Blocos & 1 & & \\
Touceira d. de Blocos & 28 & $Q_{1}$ & $V_{1}=\sigma^{2}+4 \sigma_{1}^{2}$ \\
Canas d. de Touceiras & 90 & $Q_{2}$ & $V_{2}=\sigma^{2}$ \\
\hline
\end{tabular}

$\sigma^{2}=$ variância de $e_{i j k}$;

$\sigma_{1}^{2}=$ variância de $t_{i j}$;

$\hat{\sigma}^{2}=Q_{2}=Q M$ Canas $\mathrm{d}$. Touceiras

$\hat{\sigma}_{1}^{2}=\frac{1}{4}\left(Q_{1}-Q_{2}\right)$

Mostram que uma amostra de 20 canas, no caso de 20 canas da mesma touceira, teria como variância da média:

$$
\mathrm{V}\left(\mathrm{m}_{20}\right)=\frac{\sigma^{2}+20 \sigma_{1}^{2}}{20}=\frac{5 \mathrm{~V}_{1}-4 \mathrm{~V}_{2}}{20}
$$

enquanto que com 20 canas, mas uma de cada touceira obteriam:

$$
\mathrm{V}\left(\mathrm{m}_{1}\right)=\frac{\sigma^{2}+\sigma_{1}^{2}}{20}-\frac{1}{20} \cdot \frac{\mathrm{v}_{1}+3 \mathrm{~V}_{2}}{4}
$$

Admitindo este modelo matemātico deveriam ter sempre $\mathrm{V}_{3}>\mathrm{V}_{2}$, resultando sempre em menor variância da amostra obtida com uma só cana por touceira.

No entanto, admitindo a possibilidade de ter $\mathrm{v}_{1}<\mathrm{v}_{2}$, consideraram o modelo: 


$$
y_{i j k}=m+b_{i}+e_{i j k},
$$

admitindo para duas canas da mesma touceira $\underline{j}$, um coeficiente de cor relação $\rho$, resultando na anālise de variância seguinte:

\begin{tabular}{l|c|c|c}
\hline Causas de Variação & G.L. & Q.M. & E(Q.M.) \\
\hline Blocos & 1 & & \\
Touceira d. de Blocos & 28 & $Q_{1}$ & $V_{1}=\sigma^{2}(1+3 \rho)$ \\
Canas d. de Touceiras & 90 & $Q_{2}$ & $V_{2}=\sigma^{2}(1-\rho)$ \\
\hline
\end{tabular}

No caso de ter $\rho>0$, então $v_{1}>V_{2}$, recaindo-se no caso anterior. Mas se tivesse $\rho<0$, então ficaria $v_{1}<v_{2}$. Então:

$$
\begin{gathered}
\sigma^{2}=\frac{v_{1}+3 v_{2}}{4} \\
\rho=\frac{v_{1}-v_{2}}{v_{1}+3 v_{2}}
\end{gathered}
$$

e:

$$
\begin{gathered}
\hat{\sigma}^{2}=\frac{Q_{1}+3 Q_{2}}{4} \\
\hat{\rho}=\frac{Q_{1}-Q_{2}}{Q_{1}+3 Q_{2}} .
\end{gathered}
$$

A partir desses valores mostram que, para 20 canas de uma mesma touceira: 
10.

$$
\mathrm{V}\left(\mathrm{m}_{20}\right)=\frac{\sigma^{2}(1+19 p)}{20}=\frac{5 \mathrm{~V}_{1}-4 \mathrm{~V}_{2}}{2}
$$

e para 20 canas, uma por touceira,

$$
\mathrm{V}\left(\mathrm{m}_{1}\right)=\frac{\sigma^{2}}{20}=\frac{1}{20} \cdot \frac{\mathrm{v}_{1}+3 \mathrm{v}_{2}}{4}
$$

Assim, mostram não haver influência da correlação no cálculo da variância da média.

RICO GUTIERREZ (1965) cita que, entre as anomalias que podem ocorrer ao estimar a herdabilidade como correlação entre meio-irmãos, pode acontecer que o quadrado médio para pais (machos) seja inferior ao quadrado médio para mães (fêmeas) e, por isso, a variância devida a machos seria negativa, o que teoricamente não po deria acontecer. Aconselha supor-se que a influência dos machos é nula, e agrupar estes com as fêmeas, somando os graus de liberdade e as somas de quadrados de ambos, calculando um quadrado médio correspondente a esses graus de liberdade. Isto equivaleria a supor que não existe agrupamento das fêmeas dentro dos machos.

SNEDECOR (1966) cita um exemplo em que o quadrado mé dio para amostras é menor que para sub-amostras, e admite o componente de variância para amostras com estimativa igual a zero. Discu te também sobre a correlação intra-classe, indicando como obter sua 
estimativa, e como verificar sua significância. Conclui que uma gran de correlação intra-classe indica uma variação relativamente pequena entre os individuos das sub-amostras.

BECKER (1967) apresenta o modelo matemático e a anālise de variância para o caso de classificação hierárquica com núme ros diferentes de fêmeas por macho e de filhos por fêmea.

0 modelo é:

$$
y_{i j k}=\mu+\alpha_{i}+\beta_{i j}+e_{i j k},
$$

onde:

$$
\begin{aligned}
y_{i j k}= & \text { valor da progênie } \underline{k} \text { da fêmea } \underline{j} \text { e do macho } \underline{i} ; \\
\mu= & \text { média geral } \\
\alpha_{i}= & \text { efeito do macho } \underline{i} ; \\
\beta_{i j}= & \text { efeito da fêmea } \underline{j} \text { acasalada com o macho } \underline{i} ; \\
e_{i j k}= & \text { fatores não controlados e desvios genéticos atribuídos } \\
& \text { aos indivíduos. }
\end{aligned}
$$

O esquema da anālise de variância é:

\begin{tabular}{l|c|c|c}
\hline Causas de variação & G.L. & S.Q. & E(Q.M.) \\
\hline Entre machos & $\mathrm{S}-1$ & $\mathrm{SQ}_{\mathrm{S}}$ & $\sigma_{\mathrm{W}}^{2}+\mathrm{K}_{2} \sigma_{\mathrm{D}}^{2}+\mathrm{K}_{3} \sigma_{\mathrm{S}}^{2}$ \\
Entre fêmeas d. de machos & $\mathrm{D}-1$ & $\mathrm{SQ}_{\mathrm{D}}$ & $\sigma_{\mathrm{W}}^{2}+\mathrm{K}_{1} \sigma_{\mathrm{D}}^{2}$ \\
Pro.gênies d. de fêmeas & n..-D & $\mathrm{SQ}_{\mathrm{W}}$ & $\sigma_{\mathrm{W}}^{2}$ \\
\hline
\end{tabular}

onde: $S$ = número de machos;

$$
\begin{aligned}
\mathrm{D} & =\text { nümero de fêmeas; } \\
\mathrm{n} . . & =\text { nümero total de filhos; }
\end{aligned}
$$



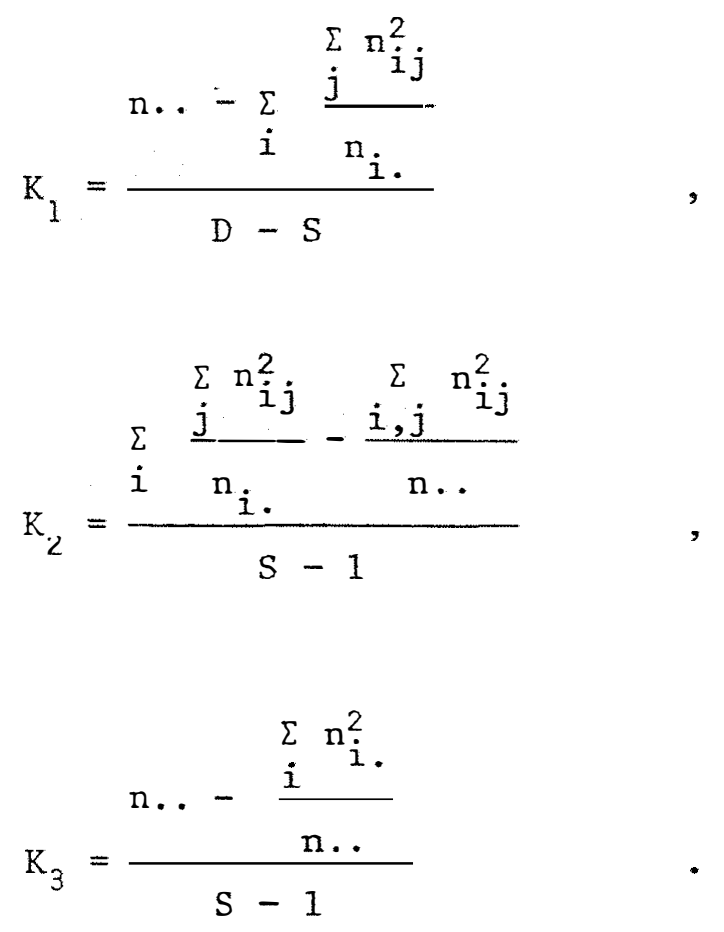

LOUCA E ROBISON (1967) não conseguiram calcular correlações genéticas entre tamanho de leitegada e outras característi cas a partir dos componentes de variância e covariância entre irmãos germanos, devido ao fato de que em todas as análises os componentes de variância para machos, para tamanho de leitegada,foi nega tivo.

KALIL (1972) encontrou duas situações em que ocorre um componente de variância negativo:

19) Para o rebanho Nelore encontrou para $\hat{\sigma}_{\mathrm{SD}}^{2}$ (Fator $\left.\mathrm{B} / \mathrm{A}\right)$, um valor de $-0,52$, que teoricamente não poderia ocorrer. Como este valor está bastante próximo de zero, admitiu-o como nu 1o. 
29) Para o rebanho Gir encontrou $\widehat{\sigma}_{\mathrm{SD}}^{2}=-183,87$. Afirma que como este componente de variância negativo é muito grande, não é indicado admiti-lo como zero. Faz uma analogia com um trabalho de PIMENTEL GOMES et alii (1963) que, estudando a amostragem da cana-de-açücar para determinaçôes tecno lógicas, onde ocorreu um problema estatístico semelhante, admitiram que, em um dos casos examinados, havia um coeficiente de correlação negativo para duas canas da mesma tou ceira. Então, concluiu que, do mesmo modo, poder-se-ia admitir um coeficiente de correlação negativo para os filhos de um mesmo casal, no caso do rebanho Gir.

Como o assunto relativo a componentes de. variância negativa é relativamente escassa, encontramos dificuldade para maio res detalhes sobre o assunto. 
Devido ao fato de existirem, na classificação hierár quica, problemas em que devemos considerar modelos aleatōrio, misto ou fixo.

E considerando o diagrama a seguir, vamos ilustrar a classificação hierārquica geral. 


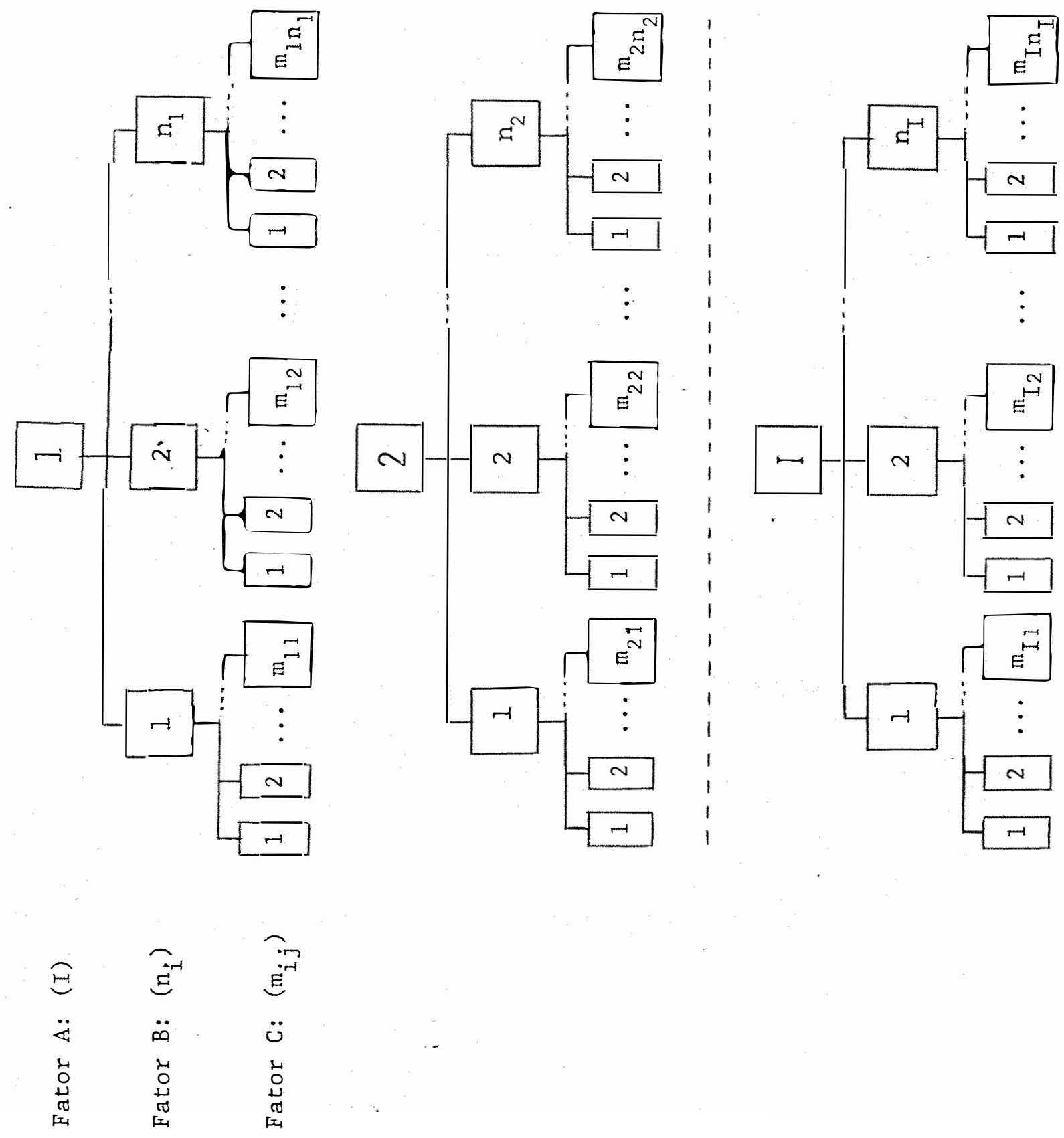


16.

e seja:

$$
\begin{aligned}
I & =\text { número de elementos do Fator } A(i=1,2, \ldots, I) ; \\
n_{i} & =\text { número de elementos do Fator } B \text { dentro de } A_{i} ; \\
m_{i j} & =\text { número de elementos do Fator } C \text { de } B_{j} \operatorname{com~} A_{i}\left(j=1,2, \ldots, n_{i}\right) ; \\
m_{i} & =\text { número total do Fator } C \text { dentro de } B_{j} ; \\
m_{.} . & =\text {número de Fator } C .
\end{aligned}
$$

\section{1 - Modelo Matemātico}

Considerando o modelo matemātico

$$
y_{i j k}=\mu+a_{i}+b_{i j}+e_{i j k} \text {, }
$$

com $k=1,2, \ldots, m_{i j}$, e onde:

$$
\begin{aligned}
& y_{i j k} \text { corresponde a uma característica do k-ésimo Fator } C \text { refe- } \\
& \text { rente ao j-ésimo Fator B dentro do i-ésimo Fator A; } \\
& \mu \text { é a média teórica; } \\
& a_{i} \text { é o efeito do Fator } A_{i} ; \\
& b_{i j} \text { é o efeito do Fator } B_{j} \text { dentro do Fator } A_{i} ; \\
& e_{i j k} \text { é o efeito do erro residual ou do Fator } C_{k} \text { dentro do Fator } \\
& \quad B \text { dentro do Fator } A_{i} \text {. }
\end{aligned}
$$




\subsection{1 - Modelo aleatōrio (Tipo II)}

Considerando-se como de efeito fixo o parâmetro $\underline{\mu} \mathrm{e}$ os demais de efeitos aleatórios, temos:

$$
\begin{aligned}
& \mathbb{E}(\mu)=\mu \quad \text { e } \quad \mathbb{E}\left(\mu^{2}\right)=\mu^{2} \\
& \mathbb{E}\left(a_{i}\right)=0 \quad \text { e } \quad \mathbb{E}\left(a_{i}^{2}\right)=\sigma_{a}^{2}, \operatorname{com} a_{i} \cap N\left(0, \sigma_{a}^{2}\right) \\
& \mathbb{E}\left(b_{i j}\right)=0 \quad \text { e } \quad \mathbb{E}\left(b_{i j}^{2}\right)=\sigma_{b}^{2}, \operatorname{com} b_{i j} \cap N\left(0, \sigma_{b}^{2}\right) \\
& \mathbb{E}\left(e_{i j k}\right)=0 \quad \text { e } \quad \mathbb{E}\left(e_{i j k}^{2}\right)=\sigma^{2}, \operatorname{com}_{i j k} \cap N\left(0, \sigma^{2}\right)
\end{aligned}
$$

\begin{tabular}{|c|c|c|c|}
\hline Causas de Variação & G.L. & Q.M. & $E(Q . M)$. \\
\hline Fator A & $I-1$ & $\mathrm{v}_{1}$ & $\sigma^{2}+k_{2} \sigma_{b}^{2}+k_{1} \sigma_{a}^{2}$ \\
\hline Fator B/A & $\sum_{i}^{I} n_{i}-I$ & $\mathrm{v}_{2}$ & $\sigma^{2}+k_{3} \sigma_{b}^{2}$ \\
\hline Fator $\mathrm{C} / \mathrm{B} / \mathrm{A}$ & m.. $-\sum_{i}^{I} n_{i}$ & $\mathrm{v}_{3}$ & $\sigma^{2}$ \\
\hline TOTAL & m.. -1 & & \\
\hline
\end{tabular}

e vamos considerar que as variāveis aleatōrias são independentes e identicamente distribuídos.

Quadro de anālise de variāncia: 
18.

onde,

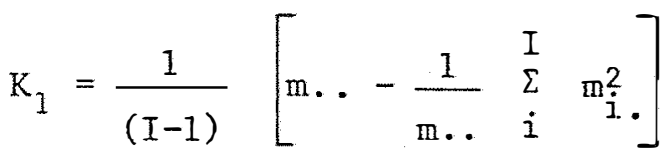

$$
\begin{aligned}
& \mathrm{K}_{2}=\frac{1}{(I-1)}\left[\sum_{i, j}^{I, n_{i}} m_{i j}^{2}\left(\frac{1}{m_{i}}-\frac{1}{m .}\right)\right]
\end{aligned}
$$

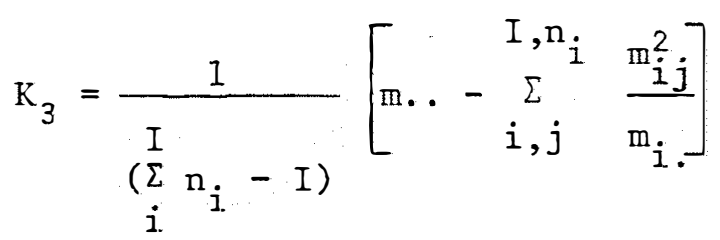

Ao fazermos a análise de variância e, observamos que $\mathrm{v}_{2}<\mathrm{v}_{3} \Longrightarrow \hat{\sigma}_{\mathrm{b}}^{2}=\left(\mathrm{v}_{2}-\mathrm{v}_{3}\right) / \mathrm{K}_{3}<0$, em primeiro lugar aplicamos o teste $\mathrm{F}$ bilateral testando a hipótese de nulidade. Temos duas situações a considerar:

a - caso não seja significativo, aceitamos a estimativa negativa, admitindo que o verdadeiro valor é zero:

b - caso seja significativo, eliminamos o parâmetro correspondente no modelo matemático.

Então o modelo $y_{i, j k}=\beta+a_{i}+b_{i j}+e_{i j k}$ passa para $y_{i, j k}=\mu+a_{i}+e_{i, j k}$, e reestruturamos a anālise de variância. Supondo, $\mathbb{E}\left[e_{i j k} \cdot e_{i} j^{\prime} k^{\prime}\right]=\left\{\begin{array}{c}\sigma^{2}, \text { para } i=i^{\prime}, j=j^{\prime} \text { e } k=k^{\prime} \\ \rho \sigma^{2}, \text { para } i=i^{\prime}, j=j^{\prime} \text { e } k \neq k^{\prime} \\ 0, \text { para os demais casos }\end{array}\right.$ sendo $\rho=$ correlação entre os elementos de $C$. 


\subsubsection{1 - Somas de quadrados}

$$
\begin{aligned}
& \text { SQ Total }=\sum_{i, j, k}^{I, n_{i}, m_{i j}} y_{i, j k}^{2}-c \quad \text {, onde } c=\frac{(y \ldots)^{2}}{m . .} \\
& \text { SQ Fator } A=\sum_{i}^{I}\left[\frac{\left(y_{i \ldots)^{2}}\right.}{m_{i}}\right]-\frac{(y \ldots)^{2}}{m . .} \\
& \text { SQ Fator } B / A=\sum_{i, j}^{I, n_{i}}\left[\frac{\left(y_{i j .}\right)^{2}}{m_{i j}}\right]-\sum_{i}^{I}\left[\frac{\left(y_{i . .}\right)^{2}}{m_{i .}}\right] \\
& \text { SQ Fator } C / B / A=\sum_{i, j, k}^{I, n_{i}, m_{i . j}} y_{i j k}^{2}-\sum_{i, j}^{I, n_{i}}\left[\frac{\left(y_{i j .}\right)^{2}}{m_{i j}}\right]
\end{aligned}
$$

e passaremos a considerar

$$
\sum_{i, j, k}^{I, n_{i j}, m_{i j}}=\sum_{i, j, k}, \sum_{i, j}^{I, n_{i}}=\sum_{i, j} \text { e } \sum_{i}^{I}=\sum_{i} .
$$

Do modelo matemātico

$$
y_{i j k}=\mu+a_{i}+e_{i . j k},
$$

$$
\sum_{k}^{m_{i j}} y_{i j k}=y_{i j .}=m_{i j} \mu+m_{i j} a_{i}+\sum_{k} e_{i j k},
$$




$$
\begin{aligned}
& \mathrm{n}_{i}, \mathrm{~m}_{i j} \\
& \underset{j, k}{\sum} y_{i j k}=y_{i . .}=m_{i}, \mu+m_{i}, a_{i}+\underset{j, k}{\sum} e_{i j k}, \\
& \sum_{i, j, k}^{I, n_{i j}, m_{i j}} y_{i j k}=y \ldots=m . . \mu+\sum_{i}^{\sum} m_{i} \cdot a_{i}+\sum_{i, j, k}^{\sum} e_{i j k},
\end{aligned}
$$

e, considerando os seguintes termos das somas de quadrados

$$
\begin{aligned}
(I) & =\frac{(y \ldots)^{2}}{m .} \\
(I I) & =\sum_{i, j, k} y_{i j k}^{2} \\
\text { (III }) & =\sum_{i, j}\left[\frac{\left(y_{i j .}\right)^{2}}{m_{i j}}\right] \\
\text { (IV) } & =\sum_{i}\left[\frac{\left(y_{i} .\right)^{2}}{m_{i}}\right]
\end{aligned}
$$

\subsubsection{2 - Determinação das esperanças matemāticas}

Utilizando o método dos momentos para a determinação das esperanças matemáticas e sendo $\mu$ constante, os termos onde o mesmo aparece se simplificam, logo não interfere na obtenção das E(QM), (esperanças dos quadrados médios corrigidos), portanto vamos suprimí-1o de todas as expressões. 
Esperança matemática da expressão (I)

$$
\begin{aligned}
& (y \ldots)^{2}=\left(\sum_{i} m_{i} . a_{i}+\sum_{i, j, k} e_{i . j k}\right)^{2} \\
& =\left(\sum_{i} m_{i} . a_{i}\right)^{2}+\left(\sum_{i, j, k} e_{i j k}\right)^{2}+d p(1)
\end{aligned}
$$

onde dp indica os duplos produtos.

$$
\begin{aligned}
& \operatorname{dp}(1)=2 \underset{i}{\sum_{i}}\left(m_{i} \cdot a_{j, k} e_{i j k}\right. \\
& \mathbb{E}[\mathrm{dp}(1)]=0 \\
& \left.\underset{i .}{\left(\sum_{i} m_{i}\right.} a_{i}\right)^{2}=\sum_{i} m_{i .}^{2} . a_{i}^{2}+d p(2) \\
& \mathrm{dp}(2)=2\left(\mathrm{~m}_{1}, \mathrm{a}_{1} \mathrm{~m}_{2} \cdot \mathrm{a}_{2}+\mathrm{m}_{1} \cdot \mathrm{a}_{1} \mathrm{~m}_{3} \cdot \mathrm{a}_{3}+\ldots+\right. \\
& +m_{I-1} \cdot a_{I-1} m_{I} \cdot a_{I} \text { ) } \\
& \mathbb{E}[\mathrm{dp}(2)]=0
\end{aligned}
$$

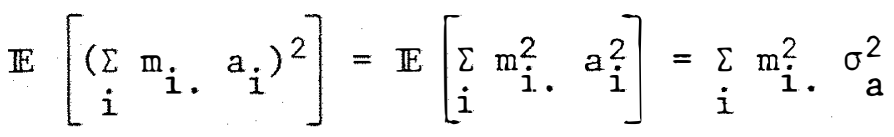




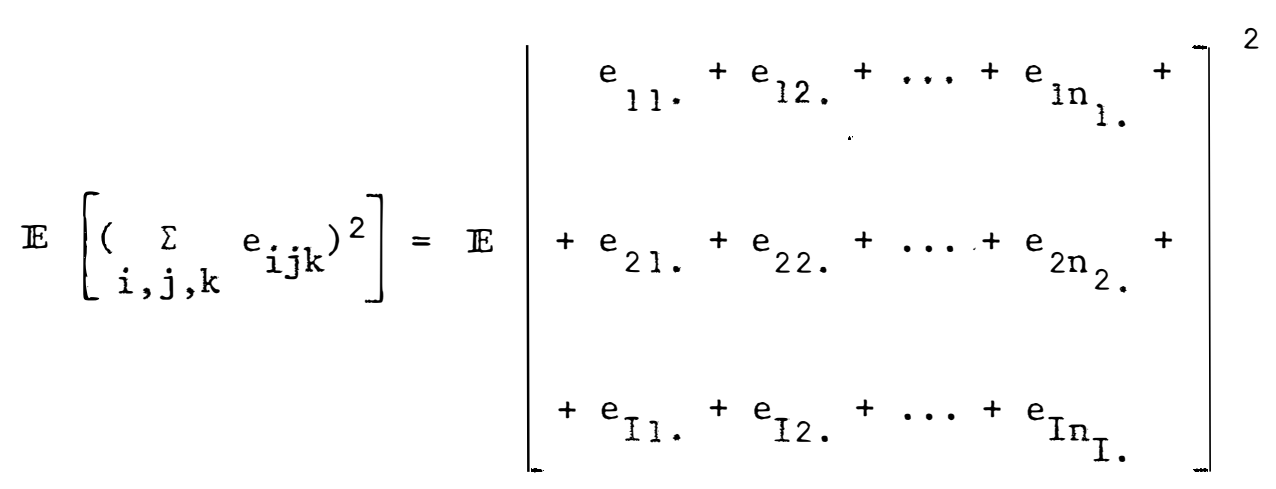

mas,

$$
\begin{aligned}
& \mathbb{E}\left(e_{i, j} \cdot e_{i \cdot{ }^{\prime} \cdot}\right)=0, \mathbb{E}\left(e_{i . j \cdot} \cdot e_{i . j}{ }^{\prime}\right)=0 \\
& \mathbb{E}\left[\left(e_{i j \cdot}\right)^{2}\right]=\mathbb{E}\left[\left(e_{i j}+e_{i j}+\ldots+e_{i j m_{i j}}\right)^{2}\right] \\
& =m_{i j} \sigma^{2}+2 \quad \sigma^{2} c_{i . j}^{2} \\
& \mathbb{E}\left[\left(\sum_{i, j} e_{i j \cdot}\right)^{2}\right]=\sum_{i, j} m_{i, j} \sigma^{2}+2 \sigma^{2} \rho \sum_{i, j} C_{i, j}^{2} \\
& =m \cdot \sigma^{2}+2 \quad \sigma^{2} \rho \sum_{i, j} \dot{C}_{i, j}^{2} \\
& 2 \sum_{i, j} C_{m_{i j}}^{2}=2 \sum_{i, j} \frac{m_{i j}\left(m_{i j}-1\right)}{2} \\
& =\underset{i, j}{\sum}\left(m_{i j}^{2}-m_{i j}\right) \\
& =\sum_{i, j} m_{i . j}^{2}-m \ldots
\end{aligned}
$$


então a Esperança Matemātica da expressão (I) será:

$$
\begin{aligned}
\mathbb{E}\left[\frac{(y \ldots)^{2}}{m . .}\right]= & \frac{\sum_{i} m_{i}^{2}}{m \ldots} \sigma_{a}^{2}+\sigma^{2}+\frac{\rho}{m \ldots}\left(\sum_{i, j} m_{i j}^{2}-m \ldots\right) \sigma^{2} \\
& =\frac{\sum_{i} m_{i}^{2}}{m \ldots} \sigma_{a}^{2}+\left[1+\frac{\rho}{m \ldots} \sum_{i, j} m_{i j}^{2}-\rho\right] \sigma^{2}
\end{aligned}
$$

$$
\mathbb{E}\left[\frac{(y \ldots)^{2}}{\mathrm{~m} . .}\right]=\frac{i^{\sum \mathrm{m}_{i}^{2}}}{\mathrm{~m} .} \sigma_{a}^{2}+\left[1+\rho(\underbrace{\mathrm{m}_{i j}^{2}}_{\mathrm{i}, j}-1)\right] \sigma^{2}
$$

Esperança matemātica da expressão (II)

$$
\begin{aligned}
\sum_{i, j, k} y_{i j k}^{2} & =\sum_{i, j, k}\left[\left(a_{i}+e_{i j k}\right)^{2}\right] \\
& =\sum_{i, j, k}\left[a_{i}^{2}+e_{i j k}^{2}+2 a_{i} e_{i j k}\right] \\
\mathbf{E}\left(\sum_{i, j, k} y_{i j k}^{2}\right) & =\underset{i, j, k}{\sum}\left[\mathbb{E}\left(y_{i j k}^{2}\right)\right] \\
& =\underset{i, j, k}{\sum}\left[\mathbb{E}\left(a_{i}^{2}+e_{i j k}^{2}+2 a_{i} e_{i j k}\right)\right] \\
& =\sum_{i, j, k} \quad\left(\sigma_{a}^{2}+\sigma^{2}\right)
\end{aligned}
$$




$$
\mathbb{E}\left(\underset{i, j, k}{\sum_{i j k}} y_{i}^{2}\right)=m . .\left(\sigma_{a}^{2}+\sigma^{2}\right)
$$

Esperança matemātica da expressão (III)

$$
\begin{aligned}
& \left(y_{i j \cdot}\right)^{2}=\left(m_{i j} a_{i}+e_{i j .}\right)^{2} \\
& =m_{i j}^{2} a_{i}^{2}+e_{i j}^{2}+2 m_{i j} a_{i} e_{i j} . \\
& \mathbb{E}\left\{\sum_{i, j}\left[\frac{\left(y_{i j .}\right)^{2}}{m_{i j}}\right]\right\}=\sum_{i, j}\left\{\mathbb{E}\left[\frac{\left.\left(y_{i j .}\right)^{2}\right]}{\left.m_{i j}\right]}\right\}\right. \\
& =\sum_{i, j}\left\{\mathbb{E}\left[\frac{1}{m_{i j}}\left(m_{i j}^{2} a_{i}^{2}+e_{i j \cdot}^{2}+2 m_{i j} a_{i} e_{i j \cdot}\right)\right]\right\} \\
& =\sum_{i, j}\left\{\frac{1}{m_{i j}}\left[\mathbb{E}\left(m_{i j}^{2} a_{i}^{2}\right)+\mathbb{E}\left(e_{i j \cdot}^{2}\right)+\mathbb{E}\left(m_{i j} a_{i} e_{i j \cdot}\right)\right]\right\} \\
& \mathbb{E}\left(m_{i j}^{2} \cdot a_{i}^{2}\right)=m_{i j}^{2} \sigma_{a}^{2} \\
& \text { De (1) temos: } \\
& \mathbf{E}\left[\left(e_{i j \cdot}\right)^{2}\right]=m_{i j} \sigma^{2}+2 \rho \sigma^{2} c_{m_{i j}}^{2},
\end{aligned}
$$


onde $2 c_{m_{i j}}^{2}=\frac{2 m_{i j}\left(m_{i j}-1\right)}{2}$

$$
\begin{aligned}
& \mathbb{E}\left[\left(e_{i j .}\right)^{2}\right]=m_{i j} \sigma^{2}+\rho m_{i j}\left(m_{i j}-1\right) \sigma^{2} \\
& =m_{i . j}\left[1+\rho\left(m_{i . j}-1\right)\right] \sigma^{2} \\
& \mathbb{E}\left(m_{i . j} a_{i .} e_{i . j}\right)=0 \\
& -\mathbb{E}\left\{\sum_{i, j}\left[\frac{\left(y_{i j .}\right)^{2}}{m_{i j}}\right]\right\}=\sum_{i, j}\left\{\frac{1}{m_{i j}}\left[m_{i j}^{2} \sigma_{a}^{2}+m_{i j}\left[1+\rho\left(m_{i j}-1\right)\right] \sigma^{2}\right]\right\} \\
& =\sum_{i, j}\left\{m_{i j} \sigma_{a}^{2}+\left[1+\rho\left(m_{i j}-1\right)\right] \sigma^{2}\right\} \\
& \mathbb{E}\left\{\sum_{i, j}\left[\frac{\left(y_{i j .}\right)^{2}}{m_{i j}}\right]\right\}=m \ldots \sigma_{a}^{2}+\left[\begin{array}{c}
\Sigma n_{i}+\rho\left(m \ldots-\sum_{i} n_{i}\right) \\
i
\end{array}\right] \sigma^{2}
\end{aligned}
$$

Esperança matemātica da expressão (IV)

$$
\begin{aligned}
\left(y_{i . .}\right)^{2} & =\left(m_{i .} a_{i}+e_{i . .}\right)^{2} \\
& =m_{i .}^{2} a_{i}^{2}+e_{i .}^{2}+2 m_{i .} a_{i} e_{i} .
\end{aligned}
$$


onde,

$$
\begin{aligned}
& \mathbb{E}\left[\begin{array}{llll}
2 \mathrm{~m}_{i} & a_{i} & e_{i} . .
\end{array}\right]=0
\end{aligned}
$$

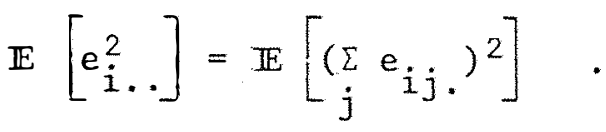

$$
\begin{aligned}
& \mathbb{E}\left[e_{i}^{2} . .\right]=\sum_{j}\left[m_{i j} \sigma^{2}+2 \rho \sigma^{2} c_{m i j}^{2}\right] \\
& =m_{i .} \sigma^{2}+2 \rho \sigma^{2} \sum_{j} C_{m i . j}^{2} \\
& =m_{i .} \sigma^{2}+2 \rho \sigma^{2} \sum_{j}\left(m_{i j}^{2}-m_{i j}\right) \\
& \mathbb{E}\left[\begin{array}{ll}
m_{i}^{2} & a_{i}^{2} \\
i &
\end{array}\right]=m_{i}^{2} \sigma^{2} \\
& \mathbb{E}\left\{\sum_{i}\left[\frac{\left(y_{i \ldots}^{2}\right)}{m_{i}}\right]\right\}=\sum_{i}\left\{\frac{1}{m_{i}} \mathbb{E}\left[\left(y_{i} . .\right)^{2}\right]\right\} \\
& =\sum_{i .}\left\{\frac{1}{m_{i}}\left[m_{i}^{2}, \sigma_{a}^{2}+m_{i .} \sigma^{2}+\rho \sigma^{2} \sum_{j}\left(m_{i . j}^{2}-m_{i j}\right)\right]\right\} \\
& =\sum_{i}\left[m_{i} \cdot \sigma_{a}^{2}+\sigma^{2}+\rho \sigma^{2}\left(\frac{\sum m_{i \cdot j}^{2}}{m_{i}}-1\right)\right]
\end{aligned}
$$


27.

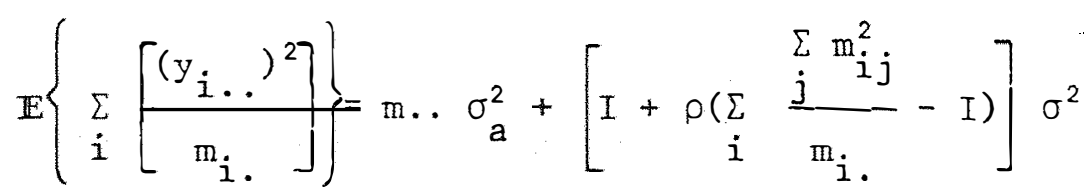

3.1.1.3 - Esperança matemática dos quadrados médios dos fatores

$$
\begin{aligned}
\mathbb{E}(\mathrm{QM} \text { Fator } \mathrm{A}) & =\frac{1}{I-1} \mathbb{E}(\mathrm{SQ} \text { Fator } \mathrm{A}) \\
& =\frac{1}{I-1}[\mathbb{E}(\operatorname{expressão} I V)-\mathbb{E}(\text { expressão } I)]
\end{aligned}
$$

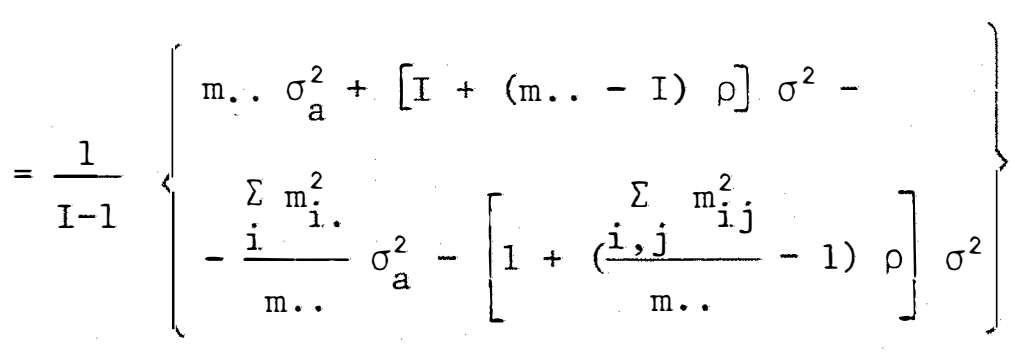

$\mathbb{E}(\mathrm{QM}$ Fator $\mathrm{A})=\frac{1}{I-1}\left[\mathrm{~m} \ldots-\frac{\sum \mathrm{m}_{i .}^{2}}{\mathrm{~m} . .}\right] \sigma_{a}^{2}+\left\{1+\left[\frac{1}{I-1}\left(\mathrm{~m} .-\frac{\sum_{i, j} \mathrm{~m}_{i, j}^{2}}{\mathrm{~m} . .}-1\right] \rho\right\} \sigma^{2}\right.$ 


$$
\begin{aligned}
& \mathbb{E}(\mathrm{QM} \text { Fator } \mathrm{B} / \mathrm{A})=\frac{1}{\sum_{i} \mathrm{n}_{i}-\mathrm{I}} \mathbb{E}(\mathrm{SQ} \text { Fator } \mathrm{B} / \mathrm{A}) \\
& =\frac{1}{\sum_{i} n_{i}-I}[\mathbb{E}(\text { expressão III })-\mathbb{E}(\text { expressão IV })] \\
& =\frac{1}{\sum_{i} n_{i}-I}\left\{\begin{array}{c}
m . . \sigma_{a}^{2}+\left[\sum_{i} n_{i}+\rho\left(m \ldots-\sum_{i} n_{i}\right)\right] \sigma^{2}- \\
-m . \sigma_{a}^{2}-\left[\begin{array}{c}
I+\rho\left(\sum \frac{j}{i} m_{i j}^{2}\right. \\
i
\end{array}\right] \sigma^{2}
\end{array}\right\} \\
& =\frac{1}{\sum_{i} n_{i}-I}\left[\left(\sum n_{i}-I\right)+\rho\left(m \ldots-\sum_{i} \frac{\sum m_{i \cdot j}}{i}-\sum_{i} n_{i}+I\right)\right] \sigma^{2}
\end{aligned}
$$$$
\mathbb{E}(\mathrm{QM} \text { Fator } \mathrm{B} / \mathrm{A})=\left\{1+\rho\left[\frac{1}{\sum_{i} \mathrm{n}_{i}-\mathrm{I}}\left(\mathrm{m}-\frac{\sum_{i} \mathrm{~m}_{i j}^{2}}{\mathrm{~m}_{i} .}-1\right]\right\} \sigma^{2}\right.
$$ 


$$
\begin{aligned}
& \left.\mathbb{E}(\mathrm{QM} C / \mathrm{B} / \mathrm{A})=\frac{1}{\mathrm{~m} .-\sum_{\mathrm{i}} \mathrm{n}_{\mathrm{i}}} \mathbb{E} \text { (SQ Fator } \mathrm{C} / \mathrm{B} / \mathrm{A}\right) \\
& =\frac{1}{\mathrm{~m} . .-\sum_{i} n_{i}}[\mathbb{E}(\operatorname{expressão} I I)-\mathbb{E}(\operatorname{expressão} \text { III })]
\end{aligned}
$$

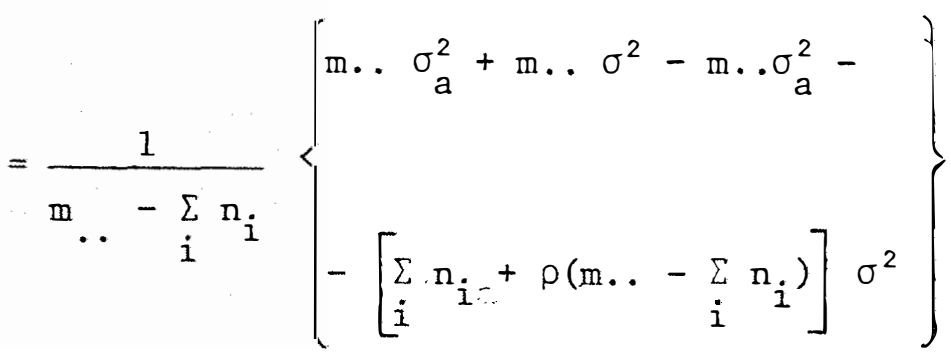

$$
\begin{aligned}
& \mathbb{E}(\mathrm{QM} C / \mathrm{B} / \mathrm{A})=(1-\rho) \sigma^{2}
\end{aligned}
$$


30.

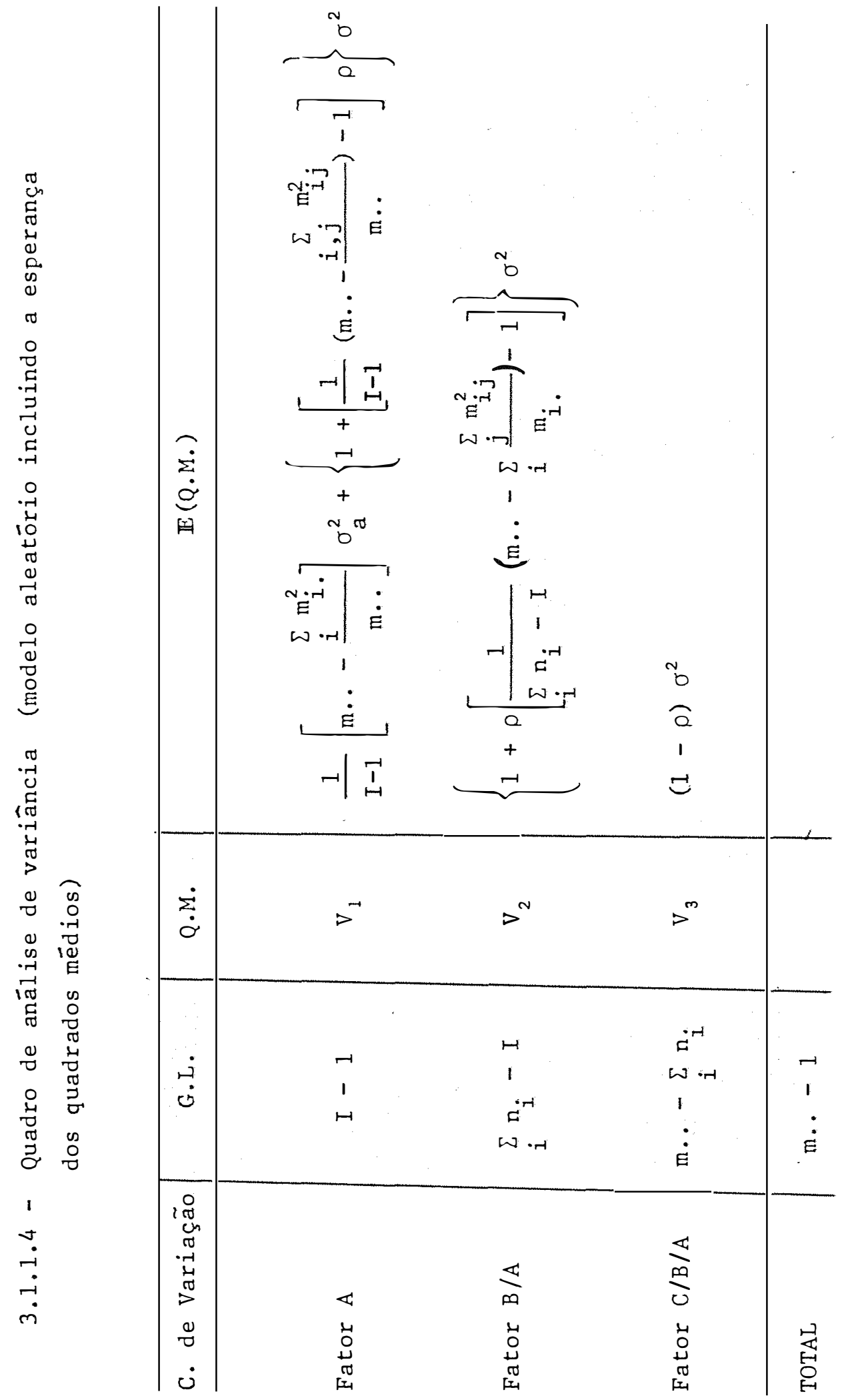




\section{1 .1 .5 - Estimativas de $\rho, \sigma_{a}^{2}$ e $\sigma^{2}$}

$$
\text { As estimativas de } \rho, \sigma_{a}^{2} \text { e } \sigma^{2} \text { são obtidas resolvendo }
$$

o sistema:

$$
\begin{aligned}
& \frac{1}{I-1}\left[m \ldots-\frac{i_{i} m_{i}^{2}}{m \ldots}\right] \hat{\sigma}_{a}^{2}+\left\{1+\left[\frac{1}{I-1}\left(m \ldots-\frac{i_{i, j}^{m_{i j}^{2}}}{m}\right)-1\right] \hat{\rho}\right\} \hat{\sigma}^{2}=V_{I} \\
& \left\{1+\hat{\rho}\left[\frac{1}{\sum n_{i}-I}\left(\mathrm{~m} . .-\sum_{i} \frac{\sum_{i j}^{2}}{m_{i}}-1\right]\right\} \hat{\sigma}^{2}=v_{2}\right. \\
& (1-\rho) \partial^{2}=V_{3}
\end{aligned}
$$

Denominando:

$$
\frac{1}{I-1}\left(\mathrm{~m} .-\frac{\sum \mathrm{m}_{i}^{2}}{\mathrm{~m} . .}\right)=a
$$

observando-se que $a=k_{1}$,

$$
\begin{gathered}
\frac{1}{I-1}\left(m \ldots-\frac{\sum_{, j}, m_{i j}^{2}}{m . .}\right)-1=b, \\
\frac{1}{\sum n_{i}-I}\left(m .-\sum_{i} \frac{\sum_{i j}^{2} m_{i}^{2}}{m_{i}}-1=c,\right.
\end{gathered}
$$


32 .

o sistema fica sendo:

$$
\left\{\begin{array}{r}
\mathrm{a} \hat{\sigma}_{\mathrm{a}}^{2}+[1+\mathrm{b} \hat{\rho}] \hat{\sigma}^{2}=\mathrm{v}_{1} \\
{[1+\mathrm{c} \hat{\rho}] \hat{\sigma}^{2}=\mathrm{v}_{2}} \\
{[1-\hat{p}] \hat{\sigma}^{2}=v_{3}}
\end{array}\right.
$$

A razão entre (3) e (4) nos dá:

$$
\hat{\rho}=\frac{v_{2}-v_{3}}{c v_{3}+v_{2}},
$$

e sendo $c v_{3}+v_{2}>0$, e, por hipótese, $v_{2}-v_{3}<0$,

$$
\hat{\rho}<0 \quad \text {. }
$$

Utilizando (3) e substituindo $\hat{\rho}$, temos:

$$
\hat{\sigma}^{2}=\frac{\mathrm{cV}_{3}+\mathrm{V}_{2}}{1+\mathrm{c}}>0
$$

Utilizando (2) e substituindo $\hat{\rho}$ e $\hat{\sigma}^{2}$, temos:

$$
\hat{\sigma}_{a}^{2}=\frac{b\left(v_{3}-v_{2}\right)+c\left(v_{1}-v_{3}\right)+\left(v_{1}-v_{2}\right)}{a(1+c)}
$$


33.

\subsubsection{6 - Valores de a, b, c}

Para que a, b e c sejam positivas, è suficiente demons

trarmos que:

$$
\sum_{i=1}^{n} a_{i}-\frac{\sum_{i=1}^{n} a_{i}^{2}}{\sum_{i=1}^{n} a_{i}}>0
$$

para $a_{i} \in N$ e pelo menos dois valores de $a_{i}$ não nulos e $i=1,2, \ldots, n$.

$$
\frac{\left(\sum_{i=1}^{n} a_{i}\right)^{2}-\sum_{i=1}^{n} a_{i}^{2}}{\sum_{i=1}^{n} a_{i}}>0
$$

$$
\left(\sum_{i=1}^{n} a_{i}\right)^{2}-\sum_{i=1}^{n} a_{i}^{2}>0
$$

$$
\sum_{i=1}^{n} a_{i}^{2}+2 \sum_{i=1}^{n} a_{i} a_{i},-\sum_{i=1}^{n} a_{i}^{2}>0, \text { com } i<i^{\prime}
$$

$$
2 \sum_{i=1}^{n} a_{i} a_{i},>0
$$




\section{1 .2 - Modelo misto (10 caso)}

Considerando o modelo misto, temos os parâmetros $\mu \mathrm{e}$ $a_{i}$ de efeitos fixos e os demais de efeitos aleatórios:

$$
\begin{aligned}
\mathbb{E}(\mu) & =\mu & \text { e } & \mathbb{E}\left(\mu^{2}\right) & =\mu^{2} \\
\mathbb{E}\left(a_{i}\right) & =a_{i} & \text { e } & \mathbb{E}\left(a_{i}^{2}\right) & =a_{i}^{2} \\
\mathbb{E}\left(b_{i j}\right) & =0 & \text { e } & \mathbb{E}\left(b_{i j}^{2}\right) & =\sigma_{b}^{2}, \operatorname{com}_{i j} \cap N\left(0, \sigma_{b}^{2}\right) \\
\mathbb{E}\left(e_{i j k}\right) & =0 & \text { e } & \mathbb{E}\left(e_{i j k}^{2}\right) & =\sigma^{2}, \operatorname{com}_{i j k} \cap N\left(0, \sigma^{2}\right)
\end{aligned}
$$

\begin{tabular}{|c|c|c|c|}
\hline Causas da Variação & G.L. & $Q \cdot M$. & $E(Q . M)$. \\
\hline Fator A & $I-1$ & $v_{1}$ & $\sigma^{2}+\mathrm{k}_{2} \sigma_{\mathrm{b}}^{2}+\mathrm{K}_{1} \phi_{\mathrm{a}}$ \\
\hline Fator B/A & $\sum_{i} n_{i}-I$ & $\mathrm{~V}_{2}$ & $\sigma^{2}+\mathrm{K}_{3} \sigma_{\mathrm{b}}^{2}$ \\
\hline Fator $C / B / A$ & m.. $-\sum_{i} n_{i}$ & $\mathrm{~V}_{3}$ & $\sigma^{2}$ \\
\hline Total & $\mathbb{R} \ldots-1$ & & \\
\hline onde, $\mathrm{K}_{1}, \mathrm{~K}_{2}$ e $\mathrm{K}_{3}$ & $\begin{array}{r}\text { são as expr } \\
\phi_{a}=\end{array}$ & $\frac{\tilde{o} \text { es } j \vec{a}}{a_{i}^{2}}$ & vistas em $(3.2 .1)$, e \\
\hline
\end{tabular}

e vamos considerar que as variáveis aleatórias são independentes e identicamente distribuidos.

Quadro de anālise de variäncia 
Ao fazermos a análise de variância e observarmos que

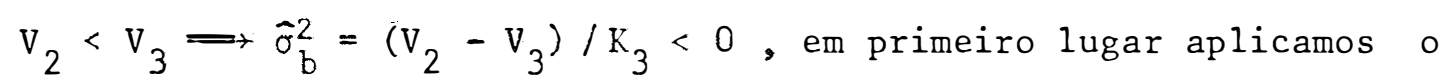
teste $F$ bilateral testando a hipótese de nulidade. Temos duas situações a considerar:

a - caso não seja significativo, aceitamos a estimativa negativa, admitindo que o verdadeiro valor é zero;

b - caso seja significativo, eliminamos o parâmetro correspondente no modelo matemático.

Então, o modelo $y_{i j k}=\mu+a_{i}+b_{i j}+e_{i j k}$ passa para $y_{i j k}=\mu+a_{i}+e_{i j k}$, e reestruturamos a análise de variância. Supondo:

$\mathbb{E}\left[e_{i j k} \cdot e_{i} j^{\prime} k^{\prime}\right]=\left\{\begin{array}{c}\sigma^{2}, \text { para } i=i^{\prime}, j=j^{\prime} \text { e } k=k^{\prime} \\ 0, \text { para } i=i^{\prime}, j=j \text {, e } k \neq k^{\prime}\end{array}\right.$ 3.T.2.1 - Somas de quadrados Consideremos as somas de quadrados $j \bar{a}$.vistas em 3.1 .1 .1 .

3.1.2.2 - Determinação das esperanças matemāticas

Sendo $u$ constante, não interfere na obtenção das E(Q.M.). Vamos suprimi-1o de todas as expressões. 
Esperança matemātica da expressão (I)

$$
\begin{aligned}
(y \ldots)^{2} & \left.=\underset{i}{\left(\sum_{i} m_{i}\right.} a_{i}+\sum_{i, j, k} e_{i j k}\right)^{2} \\
& \left.=\underset{i}{\left(\sum_{i} m_{i}\right.} a_{i}\right)^{2}+\left(\sum_{i, j, k} e_{i j k}\right)^{2}+d p(3)
\end{aligned}
$$

- le dp indica os duplos produtos.

$$
\begin{aligned}
\operatorname{dp}(3) & =2 \sum_{i}\left(m_{i}, a_{i} \sum_{j, k} e_{i j k}\right) \\
\mathbb{E}[\operatorname{dp}(3)] & =0 \\
\left.\mathbb{E}\left[\sum_{i} m_{i} \cdot a_{i}\right)^{2}\right] & \left.=\underset{i}{\left(\sum_{i} m_{i}\right.} a_{i}\right)^{2} \\
\mathbb{E}\left[\left(\sum_{i, j, k} e_{i j k}\right)^{2}\right] & \left.=m . . \sigma^{2}+\rho \sigma^{2} \underset{i, j}{\left(\sum_{i j}\right.} m^{2}-m . .\right)
\end{aligned}
$$

conforme demonstração em 3.1.1.2.

$$
\left.\left.\mathbb{E}\left[\frac{(y \ldots)^{2}}{m .}\right]=\frac{1}{m . .} \underset{i}{\left(m_{i}\right.} a_{i}\right)^{2}+\left[1+\underset{m .}{(1} \sum_{i, j} m_{i j}^{2}-1\right) \rho\right] \sigma^{2}
$$


37.

Esperança matemätica da expressão

$$
\begin{aligned}
\sum_{i, j, k}\left[y_{i j k}^{2}\right] & =\sum_{i, j, k}\left[\left(a_{i}+e_{i j k}\right)^{2}\right] \\
& =\sum_{i, j, k}\left[a^{2}+e_{i j k}^{2}+2 a_{i} e_{i j k}\right] \\
\mathbb{E}\left[\sum_{i, j, k}\left(y_{i j k}^{2}\right)\right] & =\sum_{i, j, k}\left[\mathbb{E}\left(y_{i j k}^{2}\right)\right] \\
& =\sum_{i, j, k}\left[\mathbb{E}\left(a_{i}+e_{i j k}+2 a_{i} e_{i j k}\right)\right] \\
& =\sum_{i, j, k}\left(a_{i}^{2}+\sigma^{2}\right)
\end{aligned}
$$$$
\mathbb{E}\left[\sum_{i, j, k}\left(y_{i j k}^{2}\right)\right]=\sum_{i} m_{i} \cdot a_{i}^{2}+m \ldots \sigma^{2}
$$ 
38.

\section{Esperança matemática da expressão (III)}

$$
\begin{aligned}
\left(y_{i j .}\right)^{2} & =\left(m_{i j} a_{i}+\sum_{k} e_{i j k}\right)^{2} \\
& =m_{i j}^{2} a_{i}^{2}+\left(\sum_{k} e_{i j k}\right)^{2}+2 m_{i j} a_{i} \sum_{k} e_{i j k}
\end{aligned}
$$

$$
\begin{aligned}
& \mathbb{E}\left(2 m_{i j} a_{i} \sum_{k} e_{i j k}\right)=0 \\
& \mathbb{E}\left[\begin{array}{cc}
\left(\sum_{k}\right. & \left.e_{i j k}\right)^{2}
\end{array}\right]=m_{i j} \sigma^{2}+2 \rho \sigma^{2} C_{m_{i j}}^{2} \\
& =m_{i j} \sigma^{2}+\rho \sigma^{2}\left[m_{i j}\left(m_{i j}-1\right)\right]
\end{aligned}
$$

$$
\mathbb{E}\left(m_{i j} a_{i}\right)=m_{i j}^{2} a_{i}^{2}
$$

$$
\begin{aligned}
\mathbb{E}\left\{\sum_{i, j}\left[\frac{\left.\left(y_{i j .}\right)^{2}\right]}{m_{i j}}\right]\right\} & =\sum_{i, j}\left\{\mathbb{E}\left[\frac{\left(y_{i j .}\right)^{2}}{m_{i j}}\right]\right\} \\
& =\sum_{i, j}\left[m_{i j} a_{i}^{2}+\sigma^{2}+\rho \sigma^{2}\left(m_{i j}-1\right)\right] \\
& =\sum_{i} m_{i} \cdot a_{i}^{2}+\sum_{i} n_{i} \sigma^{2}+\rho \sigma^{2}\left(m . .-\sum_{i} n_{i}\right)
\end{aligned}
$$

$$
E\left\{\sum_{i, j}\left[\frac{\left(y_{i j}\right)^{2}}{m_{i j}}\right]\right\}=\sum_{i} m_{i} \cdot a_{i}^{2}+\left[\sum_{i} n_{i}+\rho\left(m . .-\sum_{i} n_{i}\right)\right] \sigma^{2}
$$


Esperança matemática da expressão (IV)

$$
\begin{aligned}
& \left(y_{i . . .}\right)^{2}=\left(m_{i} \cdot a_{i}+\sum_{j, k} e_{i . j k}\right)^{2} \\
& =m_{i \cdot}^{2} a_{i}^{2}+\left(\sum_{j, k} e_{i j k}\right)^{2}+2 m_{i} \cdot a_{i \cdot} \sum_{j, k} e_{i j k} \\
& \mathbb{E}\left(2 \mathrm{~m}_{i} \cdot a_{i} \sum_{j, k} e_{i j k}\right)=0 \\
& \mathbb{E}\left(\sum_{j, k} e_{i j k}\right)^{2}=m_{i} . \sigma^{2}+2 \rho \sigma^{2} \sum_{j} c_{m_{i j}}^{2} \\
& =m_{i .} \sigma^{2}+\rho \sigma^{2}\left[\sum_{j}\left(m_{i j}^{2}-m_{i .}\right)\right] \\
& \mathbb{E}\left(m_{i}^{2}, a_{i}^{2}\right)=m_{i}^{2} . a_{i}^{2} \\
& \mathbb{E}\left\{\sum_{i}\left[\frac{\left(y_{i} .\right)^{2}}{m_{i}}\right]\right\}=\sum_{i}\left\{\mathbb{E}\left[\frac{\left(y_{i} .\right)^{2}}{m_{i}}\right]\right\} \\
& \left.=\sum_{i}\left[m_{i} \cdot a_{i}^{2}+\sigma^{2}+\rho \sigma^{2} \frac{\sum m_{i j}^{2}}{m_{i .}}-1\right)\right]
\end{aligned}
$$$$
\mathbb{E}\left\{\sum_{i}\left[\frac{\left(y_{i} .\right)^{2}}{m_{i}}\right]\right\}=\sum_{i} m_{i .} a_{i}^{2}+\left[I+\rho\left(\sum_{i} \frac{\sum_{i j} m_{i j}}{m_{i}}-I\right)\right] \sigma^{2}
$$ 


\subsubsection{3 - Esperança matemātica dos quadrados mēdios dos fatores}

$$
\mathbb{E}(\mathrm{QM} \text { Fator } \mathrm{A})=\frac{1}{\mathrm{I}-1}(\mathrm{SQ} \text { Fator } \mathrm{A})
$$

$$
=\frac{I}{I-1}[\mathbb{E}(\text { expressão IV) }-\mathbb{E}(\operatorname{expressão} I)]
$$

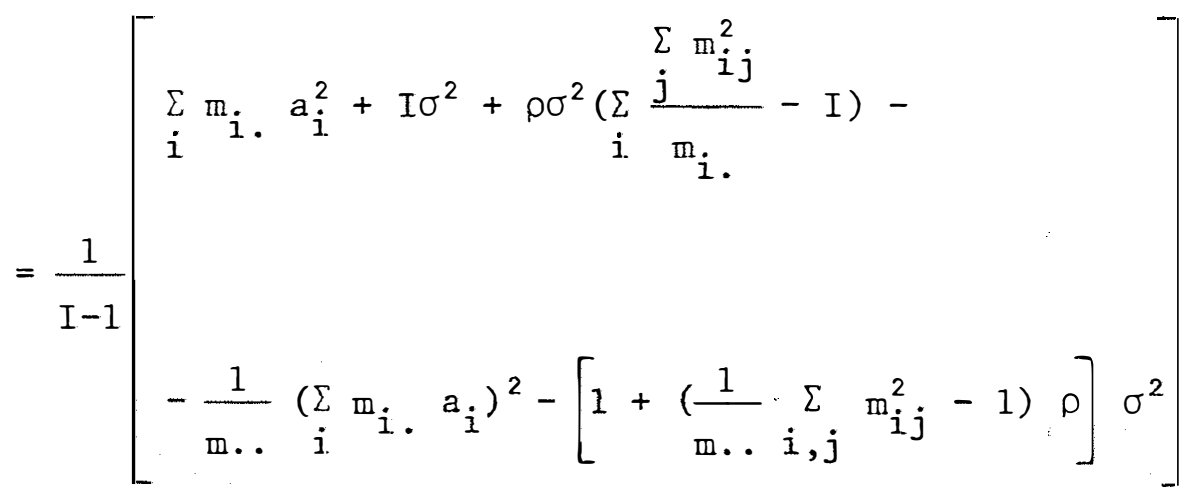

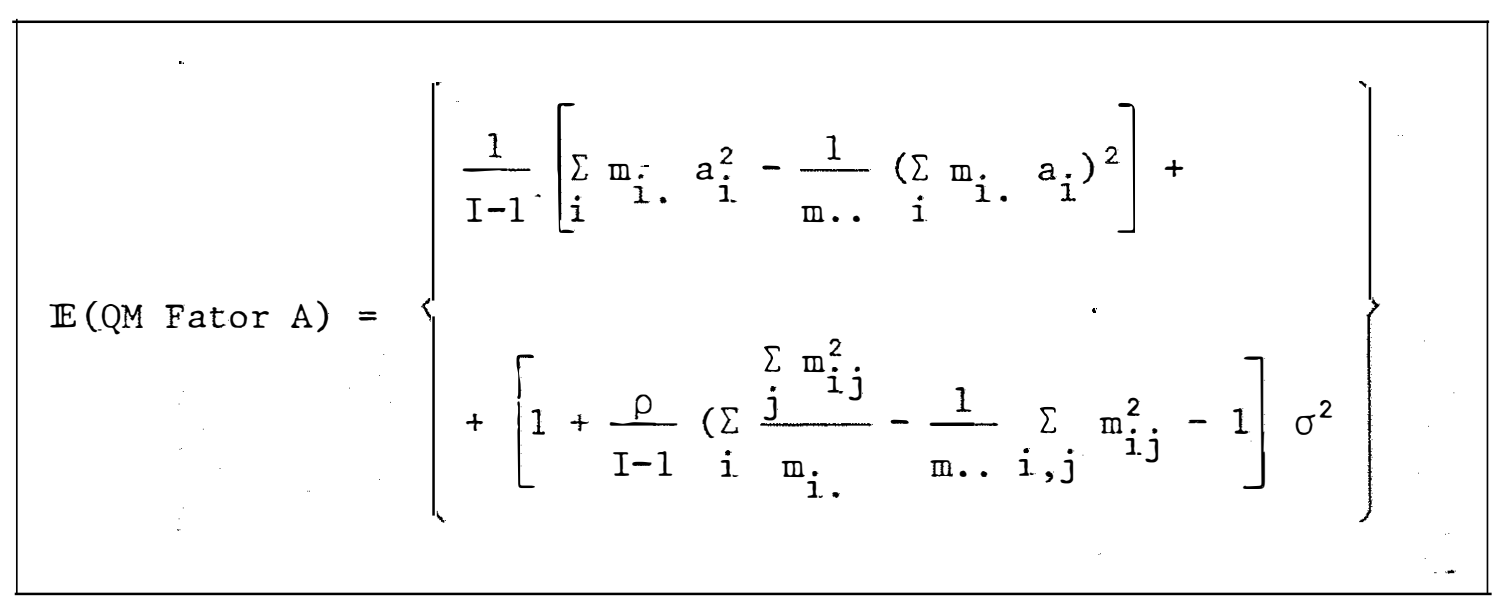




$$
\begin{aligned}
& \mathbb{E}(\mathrm{QM} \text { Fator } \mathrm{B} / \mathrm{A})=\frac{1}{\sum_{i} \mathrm{n}_{i}-I}[\mathbb{E}(\mathrm{SQ} \text { Fator } \mathrm{B} / \mathrm{A})] \\
& =\frac{1}{\sum_{i} \mathrm{n}_{i}-I}[\mathbb{E}(\operatorname{expressão} I I I)-\mathbb{E}(\text { expressão IV })] \\
& =\frac{1}{\sum_{i} n_{i}-I}\left[\begin{array}{c}
\left.\sum_{i} m_{i} a_{i}^{2}+\left[\begin{array}{l}
\sum n_{i}+\left(m . .-\sum_{i} n_{i}\right) \\
i
\end{array}\right] \sigma^{2}-\right] . \\
-\sum_{i} m_{i .} a_{i}^{2}-\left[\begin{array}{c}
I+\rho\left(\sum_{i}^{j} \frac{j m_{i j}^{2}}{m_{i .}}-I\right)
\end{array}\right] \sigma^{2}
\end{array}\right] .
\end{aligned}
$$

$$
\mathbb{E}(\mathrm{QM} \text { Fator } \mathrm{B} / \mathrm{A})=\left\{1+\rho\left[\frac{1}{\sum_{i} \mathrm{n}_{i}-\mathrm{I}}\left(\mathrm{m} . .-\sum_{i} \frac{\sum_{\mathrm{j}} \mathrm{m}_{i j}^{2}}{\mathrm{~m}_{i}}\right)-1\right]\right\} \sigma^{2}
$$

$\mathbb{E}(\mathrm{QM}$ Fator $\mathrm{C} / \mathrm{B} / \mathrm{A})=\frac{1}{\mathrm{~m} . .-\sum_{\mathrm{i}} \mathrm{n}_{i}}[\mathbb{E}($ expressão II $)-\mathbb{E}($ expressão III $)]$

$$
=\frac{1}{m \ldots-\sum_{i} n_{i}}\left[\begin{array}{l}
\sum_{i} m_{i} \cdot a_{i}^{2}+m \cdot \sigma^{2}+ \\
-\sum_{i} m_{i} \cdot a_{i}^{2}-\left[\sum_{i} n_{i}+\rho\left(m . .-\sum_{i} n_{i}\right)\right] \sigma^{2}
\end{array}\right]
$$

$$
\mathbb{E}(\mathrm{Q} M \text { Fator } \mathrm{C} / \mathrm{B} / \mathrm{A})=(1-\rho) \sigma^{2}
$$




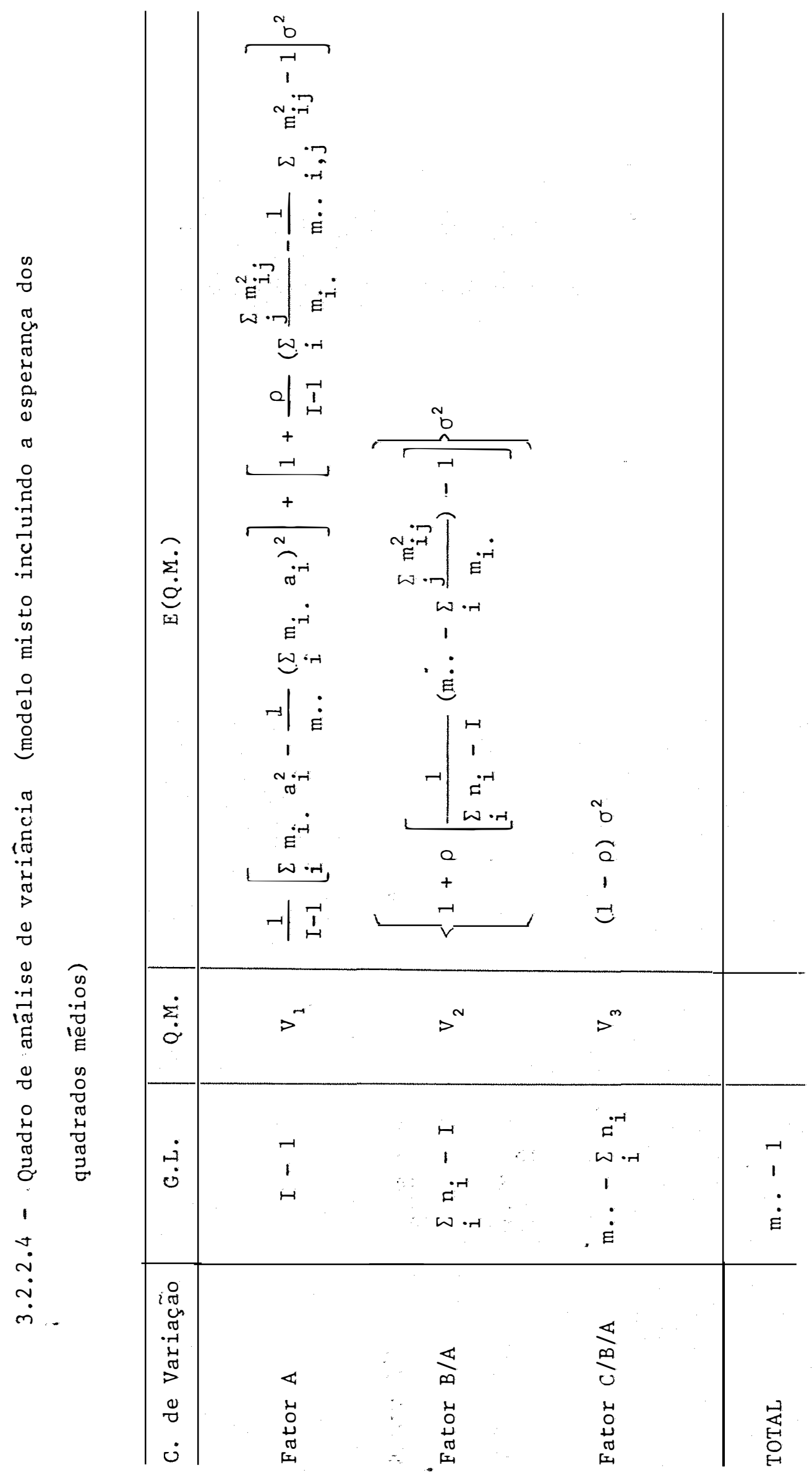


43.

3.2.2.5 - Estimativas de $\rho$ e $\sigma^{2}$

As estimativas de $\rho$ e $\sigma^{2}$ são obtidas resolvendo o sis

tema:

$$
\left\{\begin{array}{r}
\left\{1+\hat{\rho}\left[\frac{1}{\left[\sum_{i}-I\right.}\left(m \ldots-\sum_{i} \frac{\sum m_{i j}^{2}}{m_{i}}\right)-1\right]\right\} \hat{\sigma}^{2}=v_{2} \\
(1-\hat{\rho}) \hat{\sigma}^{2}=v_{3}
\end{array}\right.
$$

Denominando:

$$
\frac{1}{\sum_{i} n_{i}-I}\left(m . .-\sum_{i} \frac{\sum_{i j} m_{i j}^{2}}{m_{i}}\right)-1=a_{1}
$$

o sistema fica sendo:

$$
\left\{\begin{array}{r}
\left(1+a_{1} \hat{\rho}\right) \hat{\sigma}^{2}=v_{2} \\
(1-\rho) \hat{\sigma}^{2}=v_{3}
\end{array}\right.
$$

Efetuando a razão entre (5) e (6):

$$
\begin{gathered}
\frac{1+a_{1} \hat{\rho}}{1-\hat{\rho}}=\frac{v_{2}}{v_{3}} \\
\hat{\rho}=\frac{v_{2}-v_{3}}{a_{1} v_{3}+v_{2}}
\end{gathered}
$$


e sendo $a_{1} v_{3}+v_{2}>0$, e, por hipótese, $v_{2}-v_{3}<0$,

$$
\hat{\rho}<0 .
$$

Utilizando (6) e substituindo $\hat{p}$, temos:

$$
\sigma^{2}=\frac{a_{1} V_{3}+V_{2}}{a_{1}}
$$

ou

$$
\sigma^{2}=v_{3}+\frac{v_{2}}{a_{1}}>0 \text {. }
$$

Para que $a_{1}$ seja > 0 , observamos a demonstração

3.1 .1 .6 .

3.1 .3 - Modelo misto (20 caso)

Considerando o modelo misto, temos os parâmetros $\mu \mathrm{e}$ $b_{i j}$ de efeitos fixos, e os demais de efeitos aleatórios:

$$
\begin{array}{rlrlrl}
\mathbb{E}(\mu) & =\mu & \text { e } & \mathbb{E}\left(\mu^{2}\right) & =\mu^{2} \\
\mathbb{E}\left(\mathrm{a}_{i}\right) & =0 & \text { e } & \mathbb{E}\left(a_{i}^{2}\right) & =\sigma_{a}^{2}, \operatorname{com} a_{i} \cap N\left(0, \sigma_{a}^{2}\right) \\
\mathbb{E}\left(b_{i j}\right) & =b_{i j} & e_{i j} & \text { e } & \mathbb{E}\left(b_{i j}^{2}\right) & =b_{i j}^{2} \\
\mathbb{E}\left(e_{i j k}\right) & =0 & \text { e } & \mathbb{E}\left(e_{i j k}^{2}\right) & =\sigma^{2}, \operatorname{com} e_{i j k} \frown N\left(0, \sigma^{2}\right)
\end{array}
$$

e vamos considerar que as variāveis aleatōrias são independentes e identicamente distribuidos. 
Quadro de anātise de variāncia

\begin{tabular}{|c|c|c|c|}
\hline Causas de Variação & G.L. & $\mathrm{Q} \cdot \mathrm{M}$. & $E(Q . M)$. \\
\hline Fator A & $I-1$ & $V_{2}$ & $\sigma^{2}+\mathrm{K}_{2} \phi_{\mathrm{b}}+\mathrm{K}_{1} \sigma_{2}^{2}$ \\
\hline Fator $\mathrm{B} / \mathrm{A}$ & $\sum_{i} n_{i}-I$ & $\mathrm{~V}_{2}$ & $\sigma^{2}+\mathrm{k}_{3} \phi_{\mathrm{b}}$ \\
\hline Fator $\mathrm{C} / \mathrm{B} / \mathrm{A}$ & $m \ldots-\sum_{i} n_{i}$ & $\mathrm{~V}_{3}$ & $\sigma^{2}$ \\
\hline TOTAL & $\mathrm{m} . .-1$ & & \\
\hline
\end{tabular}

onde $K_{1}, K_{2}$ e $K_{3}$ são as expressões jā vistas em 3.2.1, e

$$
\phi_{b}=\frac{\sum_{i, j}^{I, n_{i j}} b_{i j}}{\sum_{i}^{I} n_{i}-I} .
$$

Ao fazermos a análise de variância e observarmos que $v_{2}<v_{3} \Longrightarrow \hat{\sigma}_{b}^{2}=\left(v_{2}-v_{3}\right) / k_{3}<0$, em primeiro lugar aplicamos 0 teste $\mathrm{F}$ bilateral testando a hipótese de nulidade. Temos duas situaçöes a considerar:

a - caso não seja significativo, aceitamos a estimativa negativa, admitindo que o verdadeiro valor é zero;

b - caso seja significativo, eliminamos o parâmetro correspondente no modelo matemático.

$$
\text { Então o modelo } y_{i j k}=\mu+a_{i}+b_{i j}+e_{i j k} \text { passa pa- }
$$
ra $y_{i j k}=\mu+a_{i}+e_{i j k}$, e reestruturamos a análise de variância. 
$\mathbb{E}\left[e_{i j k} \cdot e_{i} j^{\prime} k^{\prime}\right]=\left\{\begin{array}{c}\sigma^{2}, \operatorname{para} i=i^{\prime}, j=j^{\prime} \text { e } k=k^{\prime} \\ \rho \sigma^{2}, \text { para } i=i^{\prime}, j=j^{\prime} \text { e } k \neq k^{\prime} \\ 0, \text { para os demais casos }\end{array}\right.$

3.1.3.2 - Soma de quadrados

Observamos que a estrutura do modelo misto (20 caso) coincide com a do modelo aleatório, então, as novas estimativas dos componentes de variância do modelo misto (20 caso) coincidem com as do modelo aleatório.

\subsection{4 - Modelo fixo (Tipo I)}

Considerando o modelo fixo, temos os parâmetros $\mu, a_{i}$ e $b_{i j}$ de efeitos fixos e e $e_{i j k}$ de efeito aleatório,

$$
\begin{aligned}
\mathbb{E}(\mu) & =\mu & \text { e } & \mathbb{E}\left(\mu^{2}\right) & =\mu^{2} \\
\mathbb{E}\left(a_{i}\right) & =a_{i} & \text { e } & \mathbb{E}\left(a_{i}^{2}\right) & =a_{i}^{2} \\
\mathbb{E}\left(b_{i j}\right) & =b_{i j} & \text { e } & \mathbb{E}\left(b_{i j}^{2}\right) & =b_{i j}^{2} \\
\mathbb{E}\left(e_{i j k}\right) & =0 & \text { e } & \mathbb{E}\left(e_{i j k}^{2}\right) & =\sigma^{2}, \operatorname{com} e_{i j k} \quad N\left(0, \sigma^{2}\right)
\end{aligned}
$$

e vamos considerar que as variäveis aleatórias são independentes e identicamente distribuídos. 
47.

Quadro de anāitise de variāncia

\begin{tabular}{|c|c|c|c|}
\hline Causas de Variação & G.L. & Q.M. & $\mathbb{E}(\mathrm{Q} \cdot \mathrm{M})$. \\
\hline Fator A & $I-1$ & $v_{1}$ & $\sigma^{2}+k_{2} \phi_{b}+k_{1} \phi_{a}$ \\
\hline Fator B/A & $\sum_{i}^{I} n_{i}-I$ & $\mathrm{~V}_{2}$ & $\sigma^{2}+\mathrm{K}_{3} \phi_{\mathrm{b}}$ \\
\hline Fator $\mathrm{C} / \mathrm{B} / \mathrm{A}$ & $m \ldots-\sum_{i}^{I} n_{i}$ & $\mathrm{~V}_{3}$ & $\sigma^{2}$ \\
\hline TOTAL & m.. - 1 & & \\
\hline
\end{tabular}

onde $K_{1}, K_{2}$ e $K_{3}$ são as expressões jā vistas em (3.2.1),

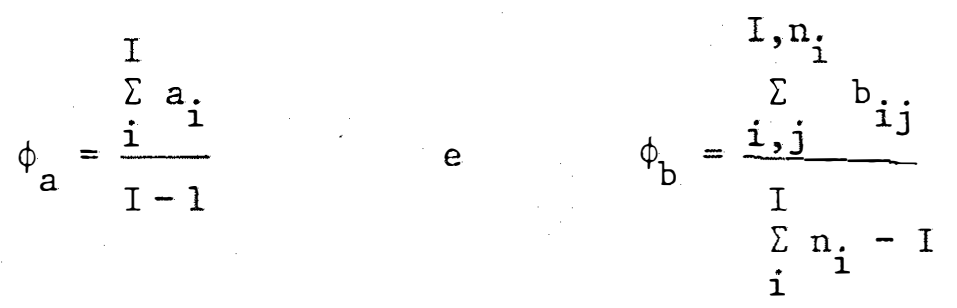

Ao fazermos a anālise de variância e observarmos que

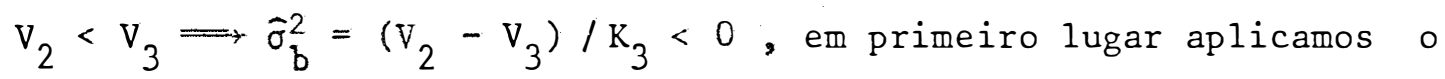
teste $\mathrm{F}$ bilateral testando a hipótese de nulidade. Temos duas situações a considerar:

a - caso não seja significativo, aceitamos a estimativa negativa, admitindo que o verdadeiro valor é zero.

b - caso seja significativo, eliminamos o parâmetro correspondente no modelo matemätico.

Então o modelo $y_{i j k}=\mu+a_{i}+b_{i j}+e_{i j k}$ passa para. $y_{i j k}=\mu+a_{i}+e_{i j k}$, e reestruturamos a anālise de variância. 
48.

Supondo:

$\mathbb{E}\left[e_{i j k} \cdot e_{i} j^{\prime} k^{\prime}\right]=\left\{\begin{array}{c}\sigma^{2}, \text { para } i=i^{\prime}, j=j \text { ' e } k=k^{\prime} \\ \rho \sigma^{2}, \text { para } i=i^{\prime}, j=j^{\prime} \text { e } k \neq k^{\prime} \\ 0, \text { para os demais casos }\end{array}\right.$

3.2.4.2 - Somas de quadrados

Observamos que a estrutura do modelo fixo coincide com a do modelo misto ( 19 caso), então as novas estimativas dos com ponentes de variância do modelo fixo coincidem com as do modelo mis to $(19$ caso) 
49.

\section{EXEMPLO}

o esquema abaixo ilustra um exemplo com dados fictícios referentes ao modelo hierárquico geral, considerando o modelo aleatörio, e supondo os valores $y_{i j k}$ com pesos em kilogramas (kg).
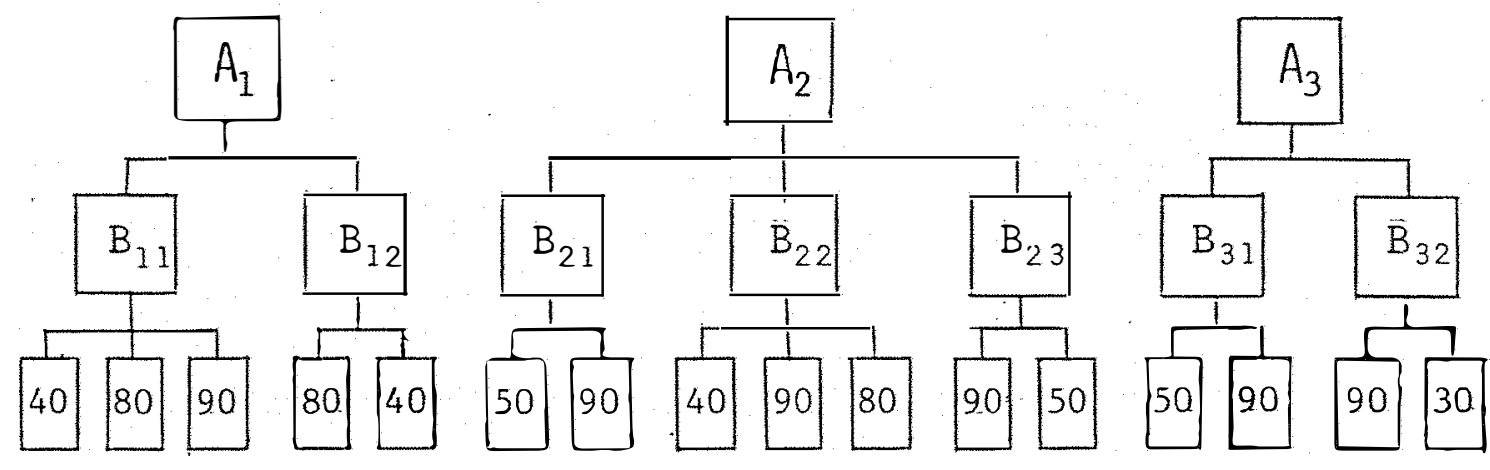
Quadro de anālise de variāncia

\begin{tabular}{l|c|c|c|c}
\hline Causas de Variação & G.L. & S.Q. & Q.M. & F \\
\hline Fator A & 2 & 0,80 & $\mathrm{~V}_{1}=0,40$ & \\
Fator B/A & 4 & 2,20 & $\mathrm{~V}_{2}=0,55$ & $0,063^{*}$ \\
Fator C/B/A & 9 & 78,00 & $\mathrm{~V}_{3}=8,67$ & \\
\hline Total & 15 & & &
\end{tabular}

Observando o quadro de análise de variância, notamos que $\mathrm{V}_{2}<\mathrm{V}_{3}$ e o teste $\mathrm{F}$ é significativo ao nível $\alpha=0,05$ de probabi lidade, portanto, justifica-se reestruturarmos a análise e, utilizando as fórmulas de 3.1.1.5, resolvendo o sistema:

$$
\begin{aligned}
a \sigma_{a}^{2}+[1+b \hat{\rho}] \hat{\sigma}^{2} & =v_{1} \\
{[1+c \hat{\rho}] \hat{\sigma}^{2} } & =v_{2} \\
{[1-\hat{\rho}] \hat{\sigma}^{2} } & =v_{3}
\end{aligned}
$$

onde,

$$
\begin{aligned}
& \mathrm{a}=\frac{1}{I-1}\left[\mathrm{~m} . .-\frac{\sum \mathrm{m}_{i}^{2} .}{\mathrm{m} . .}\right] \\
& \mathrm{b}=\frac{1}{\mathrm{I}-1}\left(\mathrm{~m} \ldots-\frac{\sum \mathrm{m}_{i j}^{2}}{\mathrm{~m} .}\right)-1
\end{aligned}
$$




$$
c=\frac{1}{\sum_{i} n_{i}-I}\left(\mathrm{~m} \cdots-\sum_{i} \frac{\sum \mathrm{m}_{i j}^{2}}{\mathrm{~m}_{i}}\right)-1
$$

onde,

$$
\begin{aligned}
& I=3 \\
& \mathrm{n}_{1}=2, \mathrm{n}_{2}=3 \text { e } \mathrm{n}_{3}=2 \Longrightarrow \sum_{\mathrm{n}^{i}} \mathrm{n}_{\mathrm{i}}=7 \\
& \mathrm{~m}_{11}=3 \text { e } \mathrm{m}_{12}=2 \Longrightarrow \mathrm{m}_{1 .}=\sum_{j=1} \mathrm{~m}_{1 j}=5 \\
& \mathrm{~m}_{21}=2, \mathrm{~m}_{22}=3 \text { e } \mathrm{m}_{23}=2 \Longrightarrow \mathrm{m}_{2 \cdot}=\sum_{j=1}^{\mathrm{n}} \mathrm{m}_{2 j}=7 \\
& m_{31}=2 \text { e } m_{32}=2 \Longrightarrow m_{3 .}=\sum_{j=1}^{n} m_{3 j}=4 \\
& {\mathrm{I}, \mathrm{n}_{\mathrm{i}}} \\
& m . .=\sum_{i, j} m_{i j}=16 \\
& \mathrm{n}_{\mathrm{i}} \\
& \sum_{i} m_{i}^{2}=m_{1}^{2}+m_{2}^{2}+m_{3}^{2} \text {. } \\
& =5^{2}+7^{2}+4^{2} \\
& n_{i} \\
& \sum_{i} \mathrm{~m}_{i}^{2}=90 \\
& I, n_{i} \\
& \sum_{i, j} m_{i j}^{2}=m_{11}^{2}+m_{12}^{2}+m_{21}^{2}+m_{22}^{2}+m_{23}^{2}+m_{31}^{2}+m_{32}^{2} \\
& =3^{2}+2^{2}+2^{2}+3^{2}+2^{2}+2^{2}+2^{2} \\
& I, n_{i} \\
& \sum_{i, j} m_{i j}^{2}=38
\end{aligned}
$$




$$
\begin{aligned}
\sum_{i} \frac{\sum_{i}^{n_{i j}} m_{i j}^{2}}{m_{i}} & =\frac{m_{11}^{2}+m_{12}^{2}}{m i .}+\frac{m^{2}+m^{2}+m_{22}^{2}}{m_{2} .}+\frac{m_{31}^{2}+m_{32}^{2}}{m_{3} .} \\
& =\frac{3^{2}+2^{2}}{5}+\frac{2^{2}+3^{2}+2^{2}}{7}+\frac{2^{2}+2^{2}}{4}
\end{aligned}
$$

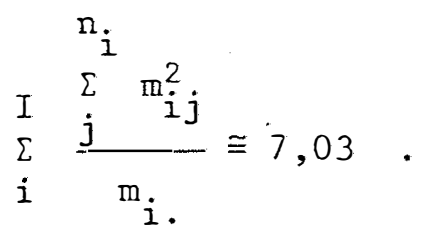

$$
\begin{aligned}
& a=\frac{1}{3-1}\left(16-\frac{90}{16}\right) \\
& a=5,1875
\end{aligned}
$$

$$
\begin{aligned}
& b=\frac{1}{3-1}\left(16-\frac{38}{16}\right)-1 \\
& b=5,8125
\end{aligned}
$$

$$
\begin{aligned}
& c=\frac{1}{7-3}(16-7,03)-1 \\
& c=1,2425
\end{aligned}
$$


53.

$$
\begin{array}{rl}
\hat{\rho} & =\frac{v_{2}-V_{3}}{c V_{3}+V_{2}} \\
\hat{\rho} & =\frac{0,55-8,67}{1,2425 \cdot 8,67+0,55} \\
\hat{\rho} & =-0,717 \\
\hat{\sigma}^{2} & =\frac{c V_{3}+v_{2}}{1+c} \\
\hat{\sigma}^{2} & =\frac{1,2425 \cdot 8,67+0,55}{1+1,2425} \\
\hat{\sigma}_{a} & 3,16 \\
\hat{\sigma}_{a} & =\frac{5\left(v_{3}-v_{2}\right)+c\left(v_{1}-v_{3}\right)+\left(v_{1}-v_{2}\right)}{2(1+c)} \\
\hat{\sigma}^{2} & =5,05
\end{array}
$$

onde obtemos uma estimativa de correlação negativa, significativa a 5\% de probabilidade, dentro do Fator C. 
54.

5. RESULTADOS OBTIDOS

Sendo $V_{1}, V_{2}$ e $V_{3}$ os quadrados médios estimados dos Fatores A, B dentro de A e C dentro de B dentro de A, respectivamen te, os estimadores dos novos componentes de variância e correlação são:

I - Para o modelo aleatório e misto (29 caso), onde foram considerados fixos os parâmetros $\mu$ e $b_{i j}$ :

$$
\begin{aligned}
& \hat{\sigma}^{2}=\frac{c v_{3}+v_{2}}{1+c}>0 \\
& \hat{\sigma}^{2}=\frac{b\left(V_{3}-v_{2}\right)+c\left(v_{1}-v_{3}\right)+\left(v_{1}-v_{2}\right)}{a(1+c)}
\end{aligned}
$$

e

$$
\hat{\rho}=\frac{v_{2}-v_{3}}{v_{3}+c v_{3}}<0 \quad \text { para } \quad v_{2}<v_{3} \text {, }
$$


sendo,

$$
\begin{aligned}
& a=\frac{1}{I-1}\left(m \ldots-\frac{\sum m_{i}^{2}}{m \ldots}\right) \\
& b=\frac{1}{I-1}\left(m . .-\frac{i, j m_{i j}^{2}}{m \ldots}-1\right. \\
& c=\frac{1}{\sum n_{i}-I}\left(m . .-\sum_{i}^{j} m_{i j}\right)-1 \\
& i m_{i}^{2}
\end{aligned}
$$

II - Para o modelo fixo e misto (10 caso) onde foram considera dos fixos os parâmetros $\mu$ e $a_{i}$

$$
\hat{\sigma}^{2}=\frac{a_{1} v_{3}+v_{2}}{a_{1}}>0
$$

e

$$
\hat{\rho}=\frac{V_{2}-V_{3}}{a_{1} V_{3}+V_{2}}<0 \quad, \quad \text { para } V_{2}<V_{3}
$$$$
\text { sendo, } a_{1}=\frac{1}{\sum_{i} n_{i}-I}\left(m . .-\sum_{i} \frac{\sum m_{i j}^{2}}{m_{i}}\right)-1 \text {. }
$$ 
56.

\section{CONCLUSÕES}

Conclui-se do presente trabalho, quando utiliza-se o modelo Classificação Hierárquica, considerando os fatores A, B dentro de $\mathrm{A}$ e $\mathrm{C}$ dentro de $\mathrm{B}$ dentro de $\mathrm{A}$, que mesmo no caso de Quadrado Médio do Fator B dentro de A ser menor que o Quadrado Médio do Fator $\mathrm{C}$ dentro de $\mathrm{B}$ dentro de $\mathrm{A}\left(\mathrm{V}_{2}<\mathrm{V}_{3}\right)$, pode-se fazer anälise de variância desde que se suponha a existência de um coeficiente decor relação inferido neste modelo.

Para tal fim, as fórmulas deduzidas neste trabalho permitiram contornar o problema de componente de variância negativa pelo método dos momentos, observando-se que o coeficiente de cor

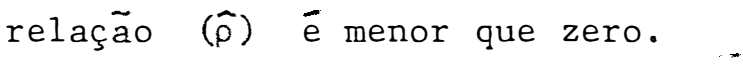


8. BIBLIOGRAFIA

ANDERSON, R.L. e T.A. BANCROFT, 1952. Statistical Theory in Research. McGraw-Hill, Nova York.

BECKER, W.A., 1967. Manual of Procedures in Quantitative Genetics. Washington State University, Pullman.

COCHRAN, W.G. e G.M. COX, 1957. Experimental Designs. 2. ed. Nova York, John Wiley \& Sons, Inc. 611 p.

FALCONER, D.S., 1960. Introduction to Quantitative Genetics. The Ronald Press Co. Nova York. 365 p.

HERBACH, L.H., 1959. Properties of Model II Type Analysis of Variance Tests. Ann. Math. Statist., 30: 939-959.

HICKS, C.R., 1973. Fundamental Concepts in the Design of Experiments. Holt, Rinehart and Winston, Nova York.

HILL, B.M., 1965. Inference about Variance Components in the OneWay Model. J.Amer. Statis. Ass., 60: 806-825.

KALIL, E.B., 1963. Repetibilidade e Hereditabilidade: um Exemplo de Aplicação. Zootecnia. Boletim no 3: 11-17. Secretaria de Agricultura. São Paulo. 
KEMPTHORNE, 0., 1957. An Introduction to Genetics Statistics. John Wiley, Nova York.

KEMPTHORNE, 0., 1965. Design and Analysis of Experiments. John Willey, Nova York.

KING, S.C. e C.R. HENDERSON, 1954. Variance components analysis in heretability studies. Poultry Science. 33: 147-154.

LOUCA, A. e O.W. ROBINSON, 1967. Components of variance and covariance in purebred and crossbred swine. J. Anim. Sci. 26: 267-273.

PANSE, V.G. e P.V. SUKHATME, 1963. Métodos Estadísticos para Investigadores Agricolas. 2a ${ }^{a}$ ed. em espanhol. México, Fondo de Cultura Econômica, 359 p.

PIMENTEL GOMES, F.; O. VALSECHI; C.P. de ABREU e E.R. de OLIVEIRA, 1964. A amostragem da cana-de-açücar para determinações tecnológicas. Anais da E.S.A. "Luiz de Queiroz", Piracicaba, 20: 89-114.

RICO GUTIERREZ, M., 1965. Genética-Estatística. Ministério de Agricultura. 195 p. Madrid.

SEARLE, S.R., 1971. Linear Models. John Wiley Inc. Nova York.

SNEDECOR, G.W., 1956. Statistical Methods Applied to Experiments in Agriculture and Biology. 5. ed. Ames, Iowa State University Press, $535 \mathrm{p}$.

SNEDECOR, G.W. e W.G. COCHRAN, 1966. Métodos Estadísticos Aplicados a la Investigación Agrícola y Biológica. 1. ed. em espanho. México, Compañia Editorial Continental S.A. 
STEEL, R.G.D. e J.H. TORRIE, 1960. Principles and Procedures of Statistics. Nova York, McGraw-Hill Book Co. 481 p.

THOMPSON, W. A. Jr., 1962. The Problem of Negative Estimates of Variance Components. Ann. Math. Statist., 33: 273-289.

TIAO, G. C. and W. Y. TAN; 1965. Bayesian Analysis of Random Effect Models in the Analysis of Variance I. Posterior Distribution of Variance-Components. Biometrika, 52: 37-53. 